\title{
A revisit to agglomerates of early-type Hipparcos stars
}

\author{
J. A. Caballero ${ }^{1, \star}$ and L. Dinis ${ }^{2}$ \\ 1 Dpto. de Astrofísica y Ciencias de la Atmósfera, Facultad de Ciencias Físicas, Universidad Complutense de Madrid, \\ E-28040 Madrid, Spain \\ 2 Grupo Interdisciplinar de Sistemas Complejos (GISC) and Dpto. de Física Atómica, Molecular y Nuclear, Universidad \\ Complutense de Madrid, E-28040 Madrid, Spain
}

Received 26 Jun 2008, accepted 30 Jun 2008

Published online .. Aug 2008

Key words stars: early-type - Galaxy: open clusters and associations: general - open clusters and associations: individual (Ori OB1b, P Puppis) - methods: data analysis - astronomical data bases: miscellaneous

\begin{abstract}
We study the spatial structure and sub-structure of regions rich in Hipparcos stars with blue $B_{T}-V_{T}$ colours. These regions, which comprise large stellar complexes, OB associations, and young open clusters, are tracers of on-going star formation in the Galaxy. The DBSCAN (Density-Based Spatial Clustering of Applications with Noise) data clustering algorithm is used to look for spatial overdensities of early-type stars. Once an overdensity, "agglomerate", is identified, we carry out a data and bibliographic compilation of their star member candidates. The actual membership in agglomerate of each early-type star is studied based on its heliocentric distance, proper motion, and previous spectro-photometric information. We identify 35 agglomerates of early-type Hipparcos stars. Most of them are associated to previously known clusters and $\mathrm{OB}$ associations. The previously unknown P Puppis agglomerate is subject of a dedicated study with Virtual Observatory tools. It is actually a new, nearby, young open cluster $(d \sim 470 \mathrm{pc}$, age $\sim 20 \mathrm{Ma})$ with a clear radial density gradient. We list P Puppis and other six agglomerates (including NGC 2451 A, vdBH 23, and Trumpler 10) as new sites for substellar searches because of their youth, closeness, and spatial density. We investigate in detail the sub-structure in the Orion, CMa-Pup and Pup-Vel OB complexes ("super-agglomerates"). We confirm or discover some stellar overdensities in the Orion complex, like the 25 Ori group, the Horsehead region (including the $\sigma$ Orionis cluster), and the $\eta$ Orionis agglomerate. Finally, we derive accurate parallactic distances to the Pleiades, NGC 2451 A, and IC 2391, describe several field early-type stars at $d<200 \mathrm{pc}$, and discuss the incompleteness of our search.
\end{abstract}

(C) 0000 WILEY-VCH Verlag GmbH \& Co. KGaA, Weinheim

\section{Introduction}

The works by Pannekoek (1929), Ambartsumian (1947) and Ruprecht (1966) were, according to de Zeeuw et al. (1999), among the most important ones for the understanding of the $\mathrm{OB}$ associations in the early pre-Hipparcos era. We also quote the comprehensive review by Blaauw (1964). OB associations are very young regions in the Galaxy containing high-mass O- and B-type stars. The ultraviolet radiation injected into the intra-association medium by these luminous blue stars play an important rôle on the subsequent lowmass star formation process. The study of OB associations is, therefore, necessary for answering critical questions in Astrophysics, like 'how does the fragmentation of a primordial molecular cloud take place', 'which is the shape of the initial mass function', 'how is the dynamical evolution at the first stages of a recently-born open cluster', 'where are the spiral arms of our Galaxy', or 'do early-type stars inhibit or facilitate the formation of substellar objects'.

After the magnus opus by de Zeeuw et al. (1999), who carried out a comprehensive census of the stellar content

\footnotetext{
* Corresponding author: José A. Caballero, investigador Juan de la Cierva at the UCM (e-mail: caballerodastrax.fis.ucm.es).
}

of nearby ( $d<1 \mathrm{kpc}$ ) OB associations, based on Hipparcos positions, proper motions, and parallaxes, and prior to launch of the European Space Agency (ESA) mission GAIA, very few "fresh" discoveries can be achieved on OB associations. The work by de Zeeuw et al. (1999) is not, however, the only search for star clusters and OB associations from the Hipparcos data: Platais, Kozhurina-Platais \& van Leeuwen (1998) and Robichon et al. (1999) had previously done it (see also a posterior work by Baumgardt, Dettbarn \& Wielen [2000]).

The superior capabilities and results of the ESA astrometry mission Hipparcos (Perryman et al. 1997) have allowed astronomers to go on the characterization of the Galactic stellar populations in general, and of $\mathrm{OB}$ associations in particular. There have been other works with the Hipparcos catalogue aimed at studying particular regions containing early-type stars (Baumgardt 1998; de Bruijne 1999; Subramaniam \& Bhatt 2000) or the Galactic structure attending to the spatial distribution of such stars (Comerón, Torra \& Gómez 1998; Maíz-Apellániz 2001; Schröder et al. 2004). We all place our trust in the "fresh" discoveries on OB associations that will arise in the near future with the overwhelming GAIA dataset. It is expected, however, that the GAIA mission will be launched in the second half of 2011. 
In the interim, we can still explore the $\mathrm{OB}$ associations with the Hipparcos catalogue and different aims, tools, and reductions of the raw data. On the one hand, in the current work, we have used the late re-reduction of the Hipparcos data by van Leeuwen (2007a), who has obtained improvements by up to a factor 4 in the astrometric accuracies for particular bright stars (see also details on the validation of the new Hipparcos reduction in van Leeuwen [2007b]). On the other hand, we have applied a novel (in Galactic astronomy) clustering analysis to part a dataset into subsets ("clusters" or "agglomerates"). Through data clustering, objects with a common distinguishing feature are classified into different groups. In our case, the common quality is just the spatial location of Hipparcos stars with blue colours (i.e. with early spectral types). That is, our aim is to study the structure (and super- and sub-structure) of Galactic OB associations, and derive some basic properties.

\section{Analysis}

\subsection{Data clustering}

\subsubsection{Types of clustering}

In short, data clustering is a common technique for statistical data analysis with the aim to classificate elements into different groups or "clusters". It is widely used in some scientific disciplines, like Computational Biology (for gene finding or plant systematics), market research (for partitioning a population of consumers into market segments), or pattern recognition in machine learning (according to the clustering illusion, the natural human "see patterns where actually none exists"). There are several types of data clustering algorithms (and classifications, as well). The simplest categorization splits the clustering algorithms into hierarchical and partitional ones; hierarchical algorithms can be, in their turn, divisive or agglomerative. The similarity of two elements within a cluster is quantified with a distance measure, that is, in most of the cases, the Euclidean distance (there are also other distances, like the Manhattan, Mahalanobis, and Hamming ones). An agglomerative hierarchical clustering algorithm begins with each element as a separate cluster and, through comparison with other elements, progessively merges them into clusters. The single linkage clustering ("nearest neighbour") is a simple agglomerative method.

\subsubsection{Clustering in Astrophysics}

Numerous astronomers and cosmologists have investigated hierarchical extragalactic clusterings (see a good example in White \& Frenk [1991]). Different algorithms to find groups, clusters, and superclusters of galaxies were early presented by, e.g., Turner \& Gott (1976), Huchra \& Geller (1982), Press \& Davis (1982), Einasto et al. (1984), Barrow, Bhavsar \& Sonoda (1985), Maddox et al. (1990), and Martínez et al. (1990). The used algorithms consisted in two-point angular correlation functions, minimal spanning trees, multifractal measures, percolations, an the well-tested friends-of-friends (FoF) algorithm. More recent clustering analyses, particularly using data from the Sloan Digital Sky Survey and FoF-like algorithms, have been carried out by Merchán \& Zandivarez (2002), Berlind et al. (2006), Weinmann et al. (2006), or Tago et al. (2008). Besides, Uchihori et al. (2000) looked for clusters of extremely high energy cosmic rays in the northern sky, which are in generally ascribed to extragalactic clusters.

At shorter distances, but still out of our Milky Way, there have been searches of OB associations using data clustering, mostly the Path Linkage Criterion, in the Magellanic System (Battinelli 1991; Bica \& Schmitt 1995; Gouliermis et al. 2000), the Andromeda galaxy (Magnier et al. 1993; Battinelli, Efremov \& Magnier 1996), and in other galaxies in the Local Group (Demers et al. 1995; Ivanov 1996; Pietrzyński et al. 2001). Although it is obvious to extrapolate the use of such automatic procedures to search for clusters in our own Galaxy, there have been very few works on this topic. First, Mel'nik \& Efremov (1995) used the list of OB stars of Blaha \& Humphreys (1989) as their input catalogue and the Path Linkage Criterion (that is a hierarchical agglomerative algorithm) to look for Galactic OB associations. Secondly, Reylé \& Robin (2002) used the same method to search for (embedded) star clusters close to the Galacic plane with the Point Source Catalogue of the DENIS survey.

The spaghetti method used by de Zeeuw et al. (1999) and Hoogerwerf \& Aguilar (1999) to identify OB associations and nearby moving groups, respectively, the 3-D and 2-D wavelet analysis carried out by Chereul, Crézé \& Bienaymé $(1998,1999)$ and Skuljan, Heranshaw \& Cottrell (1999), and other maximum likelihood approaches (Chen et al. 1997; Asiain et al. 1999) can also be considered as uncommon examples of data clustering in the Galaxy. However, all those methods require, in general, a complex mathematical apparatus and information on the star proper motion and, sometimes, age. At the long heliocentric distances where most of the OB associations are found $(d \geq 200 \mathrm{pc})$, their stellar populations are difficult no disentangle from the background solely based on proper motions, that are typically very low $\left(\mu \leq 10 \mathrm{mas} \mathrm{a}^{-1}\right)$. The data clustering algorithm that we use in this paper resembles the FoF extragalactic algorithm, that is much easier to implement, and only requires as input the 2-D spatial coordinates $(\alpha, \delta)$ of the stars. Uncertainties in the parallax determination of the farthest Hipparcos stars may prevent the use of a 3-D FoFlike algorithm (this task will be performed in the future with GAIA data).

\subsubsection{Our clustering algorithm}

In this section, we will use the term "cluster" to define the groupings of stars obtained by the algorithm, to use the standard nomenclature in data clustering. However, in the following sections, we will use the term "agglomerate" for the 

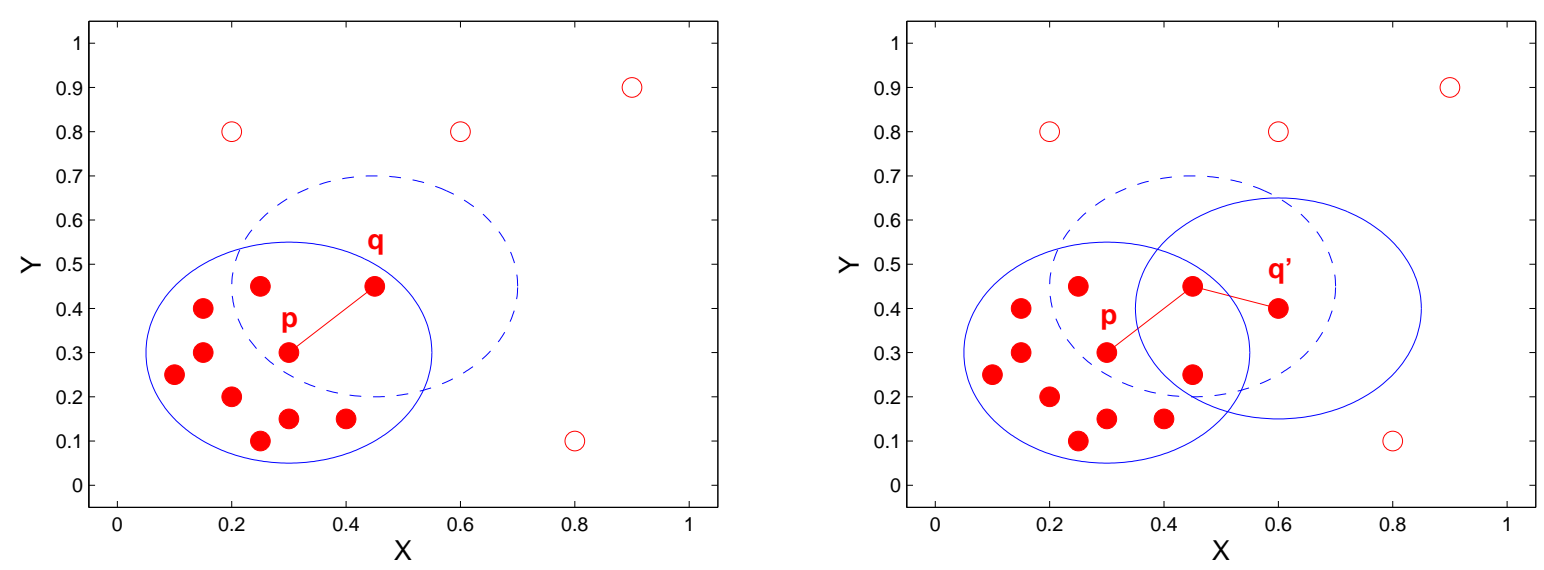

Fig. 1 A core point $p$ and a border point $q$ (left window) and a point $q^{\prime}$ density-reachable from $p$ through an intermediate (core) point (right window) for $N_{\mathrm{MinPts}}=4$ and $R_{\epsilon}=0.25$ in arbitrary units. Filled symbols represent cluster points. Colour versions of all our figures are available in the electronic publication.

different groupings of stars that the algorithm returns. This choice is to avoid confusion with the term "(star) cluster". Other expressions that we must avoid using are "(moving) group" and "(OB) association". Obviously, we expect the clustering algorithm to provide agglomerates of early-type stars that ultimately belong to a star cluster, a moving group, or an OB association, but their membership can only be confirmed after a follow-up of the individual stars.

Among the available algorithms for data clustering, we chose DBSCAN (Density-Based Spatial Clustering of Applications with Noise) for its ability to recognise clusters of arbitrary shape in a database with "noise", that is, a number of points that do not necessarily belong to any cluster. Recalling Section 2.1.1 DBSCAN is an Euclidean agglomerative hierarchical clustering algorithm. We will just outline here the fundamental aspects of density-based clustering and describe briefly the algorithm. A more detailed description of DBSCAN, including a pseudo-code, can be found in Ester et al (1996).

The notion of cluster in DBSCAN resides in the fact that the density inside a cluster is considerably higher than outside it, in the noise. This situation clearly resembles our problem, that consists in picking out densely packed groups of stars in an apparently random 2-D spatial distribution. To ensure that the clusters recognised by the algorithm are sufficiently dense, one may naïvely require that there are at least a minimum number of points $\left(N_{\mathrm{MinPts}}\right)$ in a ball of radius $R_{\epsilon}$ around every point $p$ in a cluster. A ball of radius $R_{\epsilon}$ centred on $p$ is usually termed the neighbourhood of $p$. There are, however, two kinds of points in a cluster: points well inside the cluster, or core points, and points in the border, or border points. A border point has less cluster points inside its neighbourhood than a core point, as shown in left window of Fig. 1. To include all points in a cluster, $N_{\text {MinPts }}$ should be set to a low value, what makes difficult for the algorithm to distinguish clusters from noise. To solve this difficulty, DBSCAN requires that for every point $q$ in the cluster there is another point $p$ in the cluster so that $q$ is inside the neighbourhood of $p$, and the neighbourhood of $p$ contains at least $N_{\text {MinPts }}$ points (i.e., $p$ is a core point). If $q$ is in the neighbourhood of a core point but is not a core point itself then $q$ is a border point. The following definition is central to turning this idea into an useful algorithm: a point $q$ is said density-reachable from $p$ if there is a chain of points $p=p_{1} \rightarrow p_{2} \rightarrow p_{3} \ldots p_{n}=q$ such that $p_{i+1}$ is in the neighbourhood of $p_{i}$, and the neighbourhood of $p_{i}$ contains at least $N_{\text {MinPts }}$ points. A sensible definition of a cluster is, then, the set of all points that are density-reachable from a core point, which includes all the core points as well as all the possible border points.

In short, DBSCAN starts from the first point $q$ in the database and finds all points in its neighbourhood. If $q$ is not a core point, DBSCAN tentatively marks $q$ as noise and proceeds to the next point $p$. If $p$ is a core point, all the densityreachable points from $p$ are found and marked as belonging to the same cluster. DBSCAN then proceeds to the next unclassified point in the database, repeating the procedure until all the points are marked either as noise or belonging to a cluster (i.e. an agglomerate).

\subsection{Input catalogue}

Before looking for possible agglomerates of early-type Hipparcos stars, we had to build the input dataset for our DBSCAN algorithm. By "early-type" we mean "with approximate spectral types O and B". Slightly more than $97 \%$ of the stars in the original Hipparcos catalogue $\left(N_{0}=118218\right)$ have no blanks in the parallax, proper motions, and $B_{T} V_{T}$ photometry fields, simultaneously $\left(N_{1}=114820\right)$. Among them, we selected all the sources with Tychd 1 colours $B_{T}-$ $V_{T}<-0.05 \mathrm{mag}\left(N_{2}=4142\right)$. No additional restriction was

1 For an easier analysis, we have used the Tycho-1 magnitudes (Perryman et al. 1997) instead of the slightly more precise Tycho-2 ones (Høg et al. 2000). 


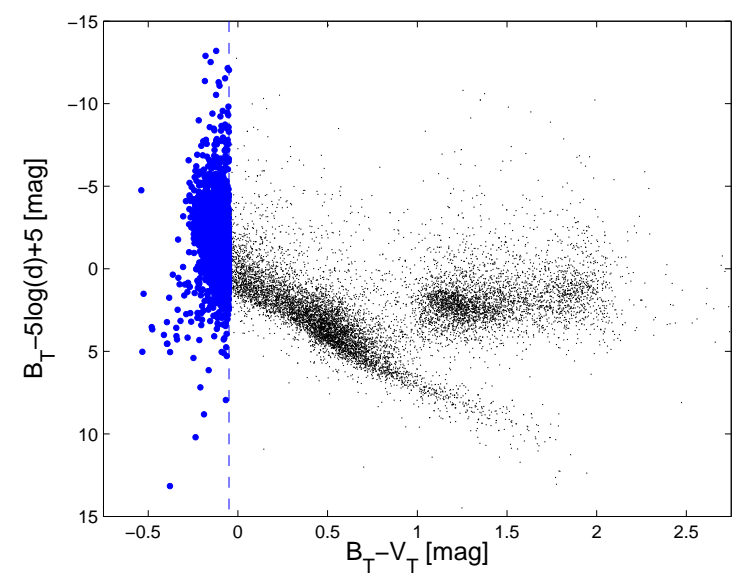

Fig. $2 B_{T}-5 \log d+5$ vs. $B_{T}-V_{T}$ diagram of the $N_{1}=114820$ Hipparcos stars with no blanks in the parallax, proper motions, and $B_{T} V_{T}$ photometry fields. It is roughly a colour-absolute magnitude $\left(M_{B_{T}}\right)$ diagram, except for the interstellar extinction factor in the $B_{T}$ band, that is not taken into account. The $N_{2}=4142$ selected stars with Tycho colours $B_{T}-V_{T}<-0.05 \mathrm{mag}$ are located to the left of the dashed vertical line.

imposed. These objects are the input for the clustering analysis described below. Fig. 2]illustrates our simple colour selection procedure.

Except for relatively high-amplitude variability, large photometric uncertainty (due to star faintness or partially resolved multiplicity), the inaccurate Johnson-Tycho systems transformation at blue colours, and the existence of white dwarfs and bright He-B subdwarfs below the main sequence, the great majority of the selected sources are Oand B-type dwarfs, giants and supergiants. The input dataset also contains a few stars with early-A spectral types; in many cases, it is due to an incorrect spectral type determination rather than to errors in the colours. Of the 11 objects with Tycho colours $B_{T}-V_{T}<-0.05 \mathrm{mag}$ and absolute magnitudes $M_{B_{T}} \approx B_{T}-5 \log d+5>5 \mathrm{mag}$, six are white dwarfs (including the intrinsically very faint, nearby, DC:-type, white dwarf GJ 440), three are B9-type dwarfs and giants with incorrect parallax determinations, one is a subdwarf (HD 188112), and one is an incorrectly identified star (BD+05 1825; see Section 4.7).

\subsection{Parameter selection}

To choose the proper parameters for the analysis, we started by setting the minimum number of bright blue stars in the cluster to a reasonable value, $N_{\text {MinPts }}=6$. Decreasing this limit would lead us to consider an unmanageable amount of agglomerates, many of which would actually be spurious groupings.

To obtain the order of magnitude of the radius $R_{\epsilon}$, we used the following heuristic argument: first, we computed the $\left(N_{\text {MinPts }}-1\right)$-distance function, mapping every point

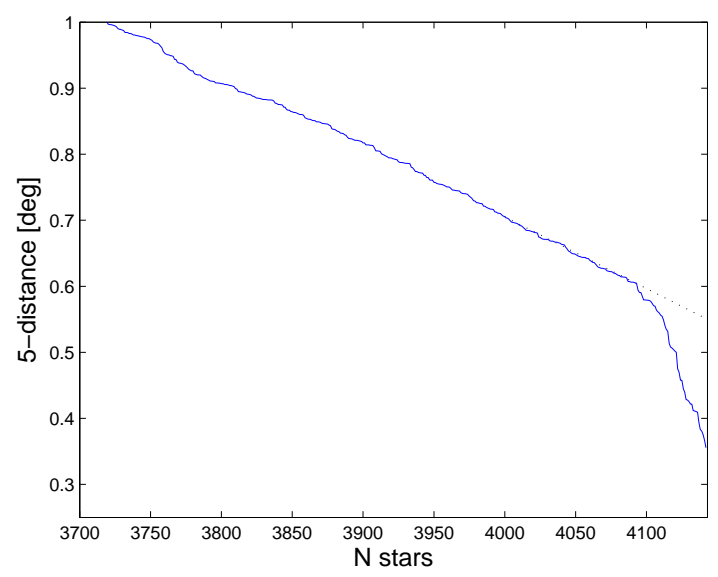

Fig. 3 Sorted 5-distance plot of the bright blue stars with the shortest 5th-distances in the input catalogue. A difference in the slope is apparent for values above and below $0.6 \mathrm{deg}$ (the dotted line extrapolates the 5-distance for $R_{\epsilon}<$ $0.6 \mathrm{deg})$.

(star) in our input catalogue with the angular distance to its fifth nearest neighbour ("5-distance"). We sorted the 5distance vector by ordering all its elements in decreasing order of their value. Fig. 3 shows a detail of the sorted 5distance, that carries information about the distribution of distances in the database (Ester et al. 1996). By setting $R_{\epsilon}$ to some fixed value, all the points (stars) with 5-distance $\leq R_{\epsilon}$ will be core points. In the figure, we see that there is a critical value at $R_{\epsilon} \sim 0.6 \mathrm{deg}$ that marks a change in behaviour. The number of points assigned to an agglomerate grows rapidly with increasing $R_{\epsilon}$ below $0.6 \mathrm{deg}$, indicating that larger increases of $R_{\epsilon}$ above that value are needed to see a significant change in the number of detected agglomerates. Once above the critical value, the results will not vary dramatically. From this, we can deduce that a value of $R_{\epsilon} \geq$ 0.6 deg should be used.

On the other hand, the DBSCAN algorithm detects aggregates with density of approximately $N_{\mathrm{MinPts}} /\left(\pi R_{\epsilon}^{2}\right)$ or higher. We used a very well known cluster, the Pleiades, as a template for computing an order of magnitude of the densities that we expect to find in our input catalogue. From the equality between densities, the neighbourhood radius is $R_{\epsilon}=R_{\text {Ple }}\left(N_{\text {MinPts }} / N_{\text {Ple }}\right)^{1 / 2}$. We have identified $N_{\text {Ple }}=$ 10 classical Pleiads as Hipparcos stars with colours $B_{T}-$ $V_{T}<-0.05 \mathrm{mag}$. All of them are contained within a $1 \mathrm{deg}-$ radius circle centred on Alcyone (i.e. $R_{\text {Ple }} \sim 1.0 \mathrm{deg}$ ), which makes $R_{\epsilon} \sim 0.8 \mathrm{deg}$. Considering this, we increased $R_{\epsilon}$ from 0.6 to $0.8 \mathrm{deg}$ in order to decrease the threshold density of the algorithm below the density of bright blue stars in the Pleiades. A discussion on the effect of the parametrization on the results (e.g. $R_{\epsilon}=0.6,0.8,1.0 \mathrm{deg}$ ) is in Section 4.6 
Table 1 Agglomerates of early-type Hipparcos stars.

\begin{tabular}{|c|c|c|c|c|c|c|c|}
\hline Agglomerate & $\begin{array}{l}\text { Reference } \\
\text { star }\end{array}$ & $\begin{array}{c}l \\
(\mathrm{deg})\end{array}$ & $\begin{array}{c}b \\
(\operatorname{deg})\end{array}$ & $\begin{array}{l}\text { Super- } \\
\text { agglomerate }\end{array}$ & $\begin{array}{l}\text { Cluster/association/ } \\
\text { complex/region }\end{array}$ & $N_{\star}$ & $\begin{array}{l}\text { Possible } \\
\text { contaminant(s) }\end{array}$ \\
\hline Escorial 1 & Alcyone & 166.7 & -43.1 & $\ldots$ & Pleiades & 10 & $\ldots$ \\
\hline Escorial 2 & V1360 Ori & 196.1 & -22.5 & Orion & $\ldots$ & 6 & (Spurious agglomerate) \\
\hline Escorial 3 & 25 Ori & 201.0 & -18.3 & Orion & Ori OB1 a, 25 Orionis & 23 & HD 35716, HD 35926 AB \\
\hline Escorial 4 & $\eta$ Ori & 204.9 & -20.4 & Orion & Ori OB 1 a, $\eta$ Orionis & 9 & HD 35957 \\
\hline Escorial 5 & $\theta^{01}$ Ori C & 209.0 & -19.4 & Orion & Ori OB1 d, Orion Nebula & 23 & $\ldots$ \\
\hline Escorial 6 & Alnilam & 205.2 & -17.2 & Orion & Ori OB1 b, Collinder 70 & 23 & $\ldots$ \\
\hline Escorial 7 & $\sigma$ Ori & 206.8 & -17.4 & Orion & Ori OB $1 \mathrm{~b}, \sigma$ Orionis & 7 & $\ldots$ \\
\hline Escorial 8 & 10 Mon & 214.5 & -7.4 & $\ldots$ & NGC 2232 & 9 & HD 45153, HD 45975 \\
\hline Escorial 9 & S Mon AB & 202.9 & +2.2 & $\ldots$ & NGC 2264, Christmas Tree & 6 & $\ldots$ \\
\hline Escorial 10 & EZ CMa & 234.8 & -10.1 & CMa-Pup & Collinder 121 & 21 & $\ldots$ \\
\hline Escorial 11 & HH CMa & 233.5 & -8.7 & CMa-Pup & Collinder 121 & 19 & $\ldots$ \\
\hline Escorial 12 & $24 \mathrm{CMa}$ & 235.6 & -8.2 & CMa-Pup & Collinder 121 & 32 & HD 53123 \\
\hline Escorial 13 & FV CMa & 236.0 & -7.3 & CMa-Pup & Collinder 121 & 7 & $\ldots$ \\
\hline Escorial 14 & HD 55019 & 240.7 & -9.0 & CMa-Pup & Collinder 121 & 6 & $\ldots$ \\
\hline Escorial 15 & $27 \mathrm{CMa} \mathrm{AB}$ & 239.0 & -7.1 & CMa-Pup & Collinder 121 & 15 & $\omega \mathrm{CMa}$ \\
\hline Escorial 16 & HD 55879 & 224.7 & +0.4 & $\ldots$ & NGC 2353 & 9 & $\ldots$ \\
\hline Escorial 17 & HD 56342 & 243.0 & -8.8 & CMa-Pup & Collinder 132 & 11 & HD 56342 \\
\hline Escorial 18 & $\tau \mathrm{CMa} \mathrm{AB}$ & 238.2 & -5.5 & CMa-Pup & Collinder 121, NGC 2362 & 16 & $\ldots$ \\
\hline Escorial 19 & NO CMa & 244.9 & -7.9 & CMa-Pup & Collinder 140 & 10 & HD 58285 \\
\hline Escorial 20 & HD 59138 & 241.0 & -4.7 & CMa-Pup & Collinder 121 & 9 & HD 59364 \\
\hline Escorial 21 & BD-14 2020 AB & 231.0 & +3.1 & $\ldots$ & M 47 & 6 & $\ldots$ \\
\hline Escorial 22 & HD 61071 & 240.4 & -2.2 & CMa-Pup & Collinder 121 & 6 & m Pup A(C)B (?) \\
\hline Escorial 23 & HD 61987 & 243.2 & -2.6 & CMa-Pup & (Collinder 121) & 8 & $\mathrm{k}^{01}$ Pup AB, HD 61687 AB \\
\hline Escorial 24 & c Pup & 252.4 & -6.7 & Pup-Vel & NGC 2451 A, NGC 2451 B & 14 & V468 Pup \\
\hline Escorial 25 & QS Pup & 260.6 & -10.4 & Pup-Vel & Vel OB2, P Puppis & 10 & HD 63007 \\
\hline Escorial 26 & $\gamma \mathrm{Vel}$ & 262.8 & -7.7 & Pup-Vel & Vel OB2, NGC 2547 & 21 & HD 67704, HD 67847, HD 68657 \\
\hline Escorial 27 & OS Pup & 254.0 & -1.0 & Pup-Vel & vdBH 23, Pup OB3 & 11 & HD 68450 \\
\hline Escorial 28 & NO Vel AB & 262.9 & -6.9 & Pup-Vel & Vel OB2 & 6 & $\ldots$ \\
\hline Escorial 29 & IT Vel & 263.4 & -6.3 & Pup-Vel & Vel OB2 & 10 & HD 70251 \\
\hline Escorial 30 & HD 72997 & 262.9 & -2.6 & Pup-Vel & vdBH 34, Pismis 4, Vel OB2 & 6 & $\ldots$ \\
\hline Escorial 31 & $o$ Vel & 270.3 & -6.8 & Pup-Vel & IC 2391 & 7 & $\ldots$ \\
\hline Escorial 32 & HX Vel & 266.6 & -3.6 & Pup-Vel & IC 2395 & 6 & HD 74273 \\
\hline Escorial 33 & HD 75387 & 262.8 & +0.7 & Pup-Vel & Trumpler 10 & 10 & $\ldots$ \\
\hline Escorial 34 & HD 194670 & 78.1 & +1.1 & $\ldots$ & "HIP 98321" & 8 & $\ldots$ \\
\hline Escorial 35 & $8 \mathrm{Lac} \mathrm{AB}$ & 96.4 & -16.1 & $\ldots$ & Lac OB1 & 6 & $\ldots$ \\
\hline
\end{tabular}

\section{Results}

Using the parameters $N_{\mathrm{MinPts}}=6, R_{\epsilon}=0.8 \mathrm{deg}$, our DBSCAN clustering algorithm has identified 406 Hipparcos stars with $B_{T}-V_{T}<-0.05$ mag distributed among $35 \mathrm{ag}-$ glomerates. These agglomerates are summarized in Table1 We follow the nomenclature Escoria 2 plus a running number to designate the agglomerates. The number of stars per agglomerate varies between $\mathrm{N}_{\star}=6$ (the minimum number) and 32. The spatial location of all the Hipparcos stars and those with blue colours are shown in top and middle windows in Fig.4.

${ }^{2}$ El Escorial, a Madritian municipality famous for its nearby Monasterio de El Escorial (a UNESCO World Heritage Site), is the hometown of both JAC and LD.
Eight of the agglomerates are "isolated", while the remaining ones seem to be associated in "super-agglomerates" (i.e. agglomerates of agglomerates). Although we have not carried out a quantitive study to distinguish super-agglomerates, they naturally arise from a simple visual inspection of the results. Besides, the existence of these super-agglomerates is endorsed by lots of previous works. One of our super-agglomerates is linked to the very famous Orion complex. The other large agglomeration of agglomerates, that occupies an extended area in the Canis Majoris-Puppis-Vela region (CMa-Pup-Vel; Kaltcheva \& Hilditch 2000), has been splitted into two differentiated super-agglomerates for simplicity. We have named them Canis Majoris-Puppis (CMa-Pup) and Puppis-Vela (Pup-Vel). The associations Ori OB1 (Orion), Collinder 121, CMa OB1, Pup OB1, and Vel OB2 (in CMa-Pup-Vel) be- 

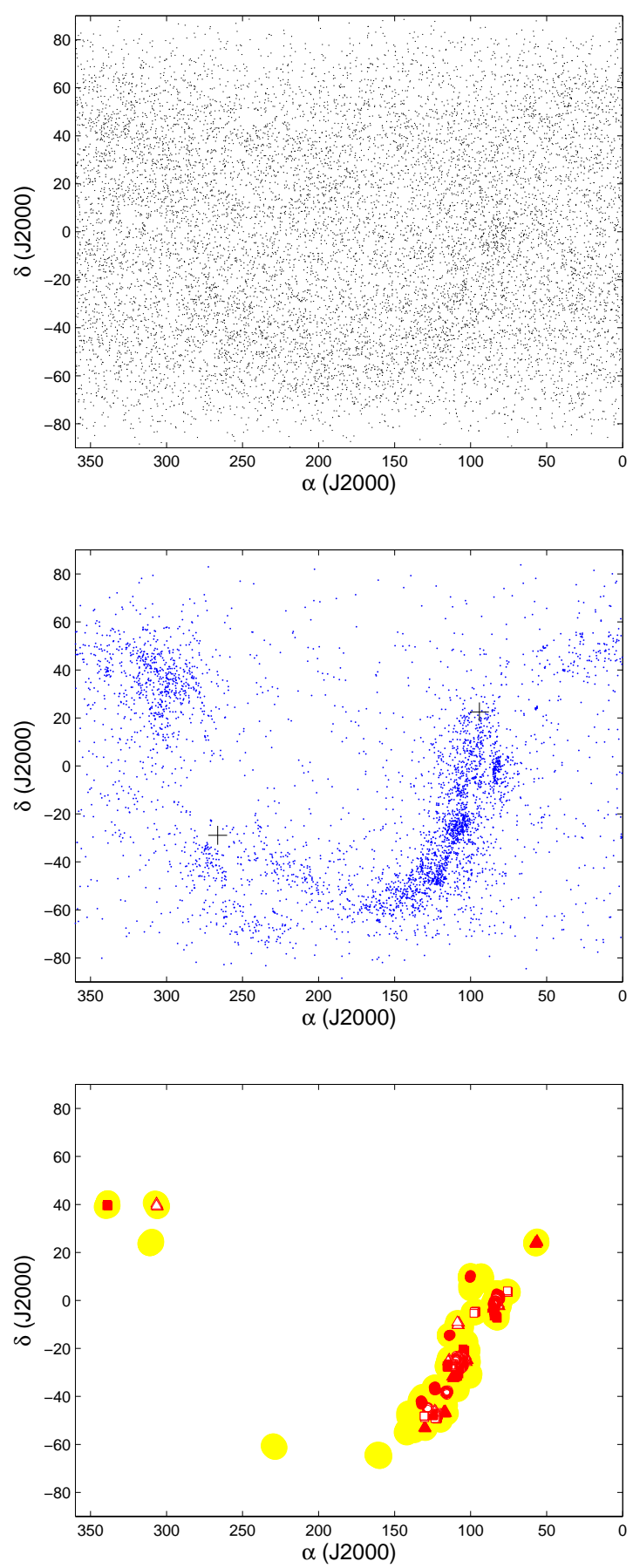

Fig. 4 Spatial distribution of early-type Hipparcos stars in agglomerates. Top window: all the $N_{1}=114820$ Hipparcos stars with no blanks in the parallax, proper motions, and $B_{T} V_{T}$ photometry fields. Middle window: $N_{2}=4142$ selected stars with Tycho colours $B_{T}-V_{T}<-0.05 \mathrm{mag}$. Crosses mark the Galactic centre (left/southeast) and anticentre (right/northwest). The plane of the Galactic disc is clearly discernible. The void of blue stars to the north of the Galactic centre is the Ophiuchus-Serpens region. Most of the apparent overdensities are discussed next. Bottom panel: blue Hipparcos stars in agglomerates with DBSCAN parameters $N_{\text {MinPts }}=6$ and $R_{\epsilon}=0.8$ (small -red-different symbols), and $N_{\mathrm{MinPts}}=6$ and and $1.0 \mathrm{deg}$ (large -yellowfilled circles). Escorial 34 (open triangles) and 35 (filled squanosjoarnathergasternmost agglomerates. long to the local spiral arm of the Galaxy (Humphreys 1978). Many of the known clusters and associations were also tabulated by Piskunov et al. (2006) as member candidates of the open cluster complex OCC 1 (which is apparently a signature of Gould's Belt).

The super-agglomerates, agglomerates and respective stellar components are described next and listed in detail in Table A1. Heliocentric distances in parenthesis are from the original Hipparcos reduction (Perryman et al. 1997), with lower eeror bars (only in these particular cases) than in van Leeuwen (2007a). Possible fore- and background blue contaminants or interlopers are also discussed. Images of the agglomerates can be found in the Appendix in the electronic version of the journal (Figs. A1 to A12).

\subsection{Isolated agglomerates}

Our clustering algorithm has identified seven agglomerates that are not ascribed to the Orion, CMa-Pup, or Pup-Vel super-agglomerates. Many of them are associated to known young open clusters or star-forming regions. Furthermore, a few of them, like the Pleiades (the template cluster for our $R_{\epsilon}$ choice), NGC 2264, or IC 2391, are among the best studied open clusters. Four of the seven agglomerates, in the Monoceros-Puppis region, are shown in Fig 5 .

\subsubsection{Escorial 1 (Pleiades)}

The Pleiades (M 45, Melotte 22, the Seven Sisters) is the open cluster par excellence. Known since antiquity, it is the most obvious cluster to the naked eye. Given its short heliocentric distance, the Pleiades stars have been widely used as calibrators for evolutionary models and as star candles (Johnson \& Mitchell 1958; Vandenberg \& Bridges 1984; Soderblom et al. 1993; Stauffer et al. 1994). The first brown dwarf, Teide 1, was discovered in this cluster (Rebolo, Zapatero Osorio \& Martín 1995).

The ten stars in the Escorial 1 agglomerate, with B68 spectral types and different classes of luminosity (III, IV, and V; see Table A1, have magnitudes, proper motions, and parallaxes consistent with membership in the Pleiades cluster. Although we have identified the ten Hipparcos stars in the region with the bluest $B_{T}-V_{T}$ colours as members of the agglomerate, they do not match the classical list of the brightest cluster stars. Absent classical Pleiads are Asterope and Celaeno, with $B_{T}-V_{T} \geq-0.05 \mathrm{mag}$, while other identified, blue, not-so-bright Pleiads are 18 Tau and HD 23753. No contaminants have been identified. See Section 4.4.1 for a discussion on the Pleiades heliocentric distance.

\subsubsection{Escorial 8 (NGC 2232)}

The NGC 2232 is a poorly known, young, open cluster in Monoceros. Its brightest members is the $\beta$ Cep-type variable star 10 Mon (B2V). Clariá (1972) carried out the first detailed photometric study of the cluster, although Herschel (1864), Dreyer (1888), and Collinder (1931) had previously 


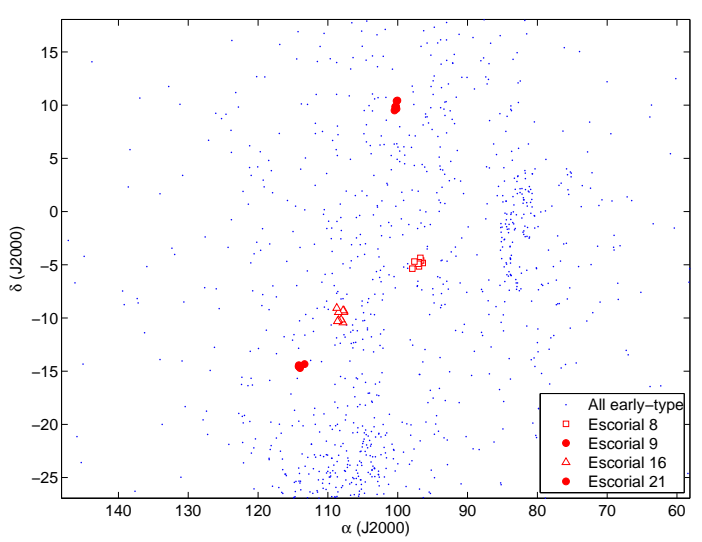

Fig.5 Spatial location of the stars of the agglomerates Escorial 8 (NGC 2232) and Escorial 9 (NGC 2264) in Monoceros, and Escorial 16 and Escorial 21 (M 17) in Puppis, in (red) different symbols (from west to east). Other early-type Hipparcos stars are marked with tiny (blue) dots. Note the overdensities corresponding to the Orion complex (at $\alpha \sim$ 80-85 deg, $\delta \sim \pm 5 \mathrm{deg}$ ) and the north of the CMa-Pup agglomerate (to the south of the plot).

catalogued it. There have been few late spectroscopic, photoelectric, and astrometric analyses (Levato \& Malaroda 1974; Pastoriza \& Ropke 1983; Jenkner \& Maitzen 1987). More recently, Lyra et al. (2006) determined nuclear and contraction ages in the narrow interval 25-32 Ma, and an heliocentric distance of $d=320 \pm 30 \mathrm{pc}$. To finish the short historical review, Dias et al. (2002) tabulated an angular diameter of about $1 \mathrm{deg}$.

Our list of nine Hipparcos stars in the Escorial 8 agglomerate roughly matches those of NGC 2232 member candidates by Robichon et al. (1999) and Baumgardt et al. (2000), as well as the brightest stars listed by Clariá (1972). The slight differences consist in the nature of a couple of possible interlopers. We have identified two contaminants, HD 45153 and HD 45975. They are two B8-9V-type stars at $d \sim 200 \mathrm{pc}$ and proper motions inconsistent with membership in cluster. The NGC 2232 cluster seems to be located, from our Hipparcos data, at $330 \pm 50 \mathrm{pc}$. This distance agrees very well with the value derived by Lyra et al. (2006) from main-sequence fitting.

\subsubsection{Escorial 9 (NGC 2264)}

The NGC 2264 cluster in the Mon OB1 association contains four structures that show clear evidence of extreme youth (age $\sim 3 \mathrm{Ma}$ ) and on-going star formation: the Christmas Tree Cluster, the Fox Fur Nebula, the Snowflake Cluster, and the Cone Nebula. Although the four structures have been considered as a single entity (precisely called NGC 2264), a purist would contradict this statement: the stellar cluster that is actually identified in the optical is the Christmas Tree Cluster, including the bright pre-main se- quence star S Mon AB (O7Ve). The latter star illuminates the nearby Fox Fur Nebula and the more distant Cone Nebula (the tip of which is just a few arcmin away from the pre-main sequence giant HD 47887 - B2III:). The nicknamed Snowflake Cluster is a dense collection of embedded, low-mass stars detected at mid-infrared and submillimetric wavelengths, adjacent to the NGC 2264 IRS2 source (Young et al. 2006), and at half distance between S Mon AB and the Cone Nebula. The "NGC 2264" cluster as a whole has been widely used to test stellar formation scenarios, measure frequencies of discs, and study $\mathrm{T}$ Tau stars and outflows (e.g. Walker 1956; Strom et al. 1972; Rebull et al. 2002; Lamm et al. 2004; Dahm \& Simon 2005).

From our clustering analysis, only six blue Hipparcos stars (the minimum number) belong to the Escorial 9 agglomerate, being S Mon AB the brightest one. Of the remaing five stars, three are relatively well known pre-mainsequence stars, and two are of unknown nature. The latter stars, HD 47754 (B9V) and HD 47662 (B7V, a lowamplitude photometric variable - Koen \& Eyer 2002), are located quite to the north of the compact $\mathrm{H}$ II region on the centre of NGC 2264. Their area is still rich in emissionline and variable stars, as well as Herbig-Haro objects, so the two early-type stars may also belong to the young starforming region.

The close separation between the components of S Mon AB ( $\left.\rho=2.909 \pm 0.013 \operatorname{arcsec}, \Delta H_{p}=3.33 \pm 0.06\right)$ may have affected the Hipparcos parallactic distance determination of the system, which is clearly incorrect $(d=$ $280 \pm 40 \mathrm{pc} ; \mathrm{S}$ Mon AB would be located at a significatively shorter heliocentric distance than the other members of NGC 2264, at about 600-800 pc).

\subsubsection{Escorial 16}

The Escorial 16 agglomerate contains nine stars. Two of them, HD 55879 and HD 55755, were classified as members of the moderately young NGC 2353 open cluster by Hoag et al. (1961). This cluster is quite compact (about $15 \mathrm{arcmin}$ ) and located near the edge of the very young CMa OB1 association (age $\sim 3 \mathrm{Ma}-$ see an excellent review on the association in de Zeeuw et al. 1999). The most comprehensive study and review of NGC 2353 to date was carried out by Fitzgerald, Harris \& Reed (1990). According to them, the cluster is at the same distance as CMa OB1 $(d=$ $1200 \pm 80 \mathrm{pc}$ ), but is several times older (age $\sim 76 \mathrm{Ma}$ ). They concluded, therefore, that NGC 2353 and CMa OB1 are unrelated, and that the brightest star in the area, HD 55879 (an early B-/late O-type giant), does not belong to the cluster, but to CMa OB1. HD 55755 would remain as the only Hipparcos star member of NGC 2353. These results are not conclusive, because Hoag et al. (1961) assumed a younger age of NGC 2353, rather similar to the age CMa OB1.

There are other seven stars at up $\sim 1.3 \mathrm{deg}$ to the NGC 2353 centre that belong to the Escorial 16 agglomerate. Only one of them is remarkable: HD 55135. It was 
one of the first emission-line, B-type (Be) stars to be discovered (Merrill, Humason \& Burwell 1925). Its strong $\mathrm{H} \alpha$ emission might indicate that it is also a few megayears old (unless Be stars are "the remnants of case B mass transfer in intermediate-mass close binaries"; Pols et al. 1991), and then HD 55135 could even be younger than NGC 2353. Of the remaining stars, HD 55117 had an Hipparcos solution flagged as unreliable (we have taken the values for its distance and proper motions from Falin \& Mignard [1999]). The other four stars have been only glanced by Clariá (1974) or have no references at all.

Obviously, further spectro-photometric analyses are needed to disentangle between the populations of NGC 2353 and CMa OB1, and to ascertain the actual nature of the Escorial 16 agglomerate. It might be a random overdensity in the disperse CMa OB1 association.

\subsubsection{Escorial 21 (M 47)}

The agglomerate Escorial 21 is associated to the young open cluster M 47 (NGC 2422 - Zug 1933; Smyth \& Nandy 1962; Shobbrook 1984; Nissen 1988; Barbera et al. 2002; Prisinzano et al. 2003). It is a $\sim 100 \mathrm{Ma}$-old cluster at $\sim 470$ pc (Rojo Arellano, Peña \& González 1997), with a minimum diameter of half a degree and a very low reddening. It has been repeatedly considered as a (more distant) Pleiades twin.

Our six stars in the Escorial 21 agglomerate are among the brightest stars of the cluster. There are only two missing bright stellar systems: the (at least) quintuple system BD142020 (the Hipparcos reduction probably failed because of the intense brightness of, and closeness between, the $\mathrm{A}$ and $\mathrm{B}$ components; $V_{A, B} \sim 6.5-7.0 \mathrm{mag}, \rho \approx 7.2 \operatorname{arcsec}$ ) and the spectroscopic binary KQ Lup AB (an A4Ia supergiant with a quite red $B_{T}-V_{T}$ colour). Our list of $\mathrm{M} 47$ member stars basically coincides with that of Robichon et al. (1999). The weight-averaged parallactic distance from the re-reduced Hipparcos stars of our six early-type stars is $d=450 \pm 100 \mathrm{pc}$, that also match with previous estimates.

\subsubsection{Escorial 34 and 35}

The 14 stars in the Escorial $34\left(\mathrm{~N}_{\star}=8\right)$ and $35\left(\mathrm{~N}_{\star}=6\right) \mathrm{ag}$ glomerates are located far away from the previous isolated clusters and from the Orion, CMa-Pup, and Pup-Vel superagglomerates. They lie in the Cygnus-Lacerta region of the Galactic plane, roughly equidistant from the North America (NGC 7000) and Cocoon (IC 5146) nebulae.

The stars of the Escorial 34 agglomerate are embedded in the $\gamma$ Cyg H II nebula (IC 1318), whose centre roughly coincides with the supergiant Sadr (F8Iab:, $V \approx 2$ mag Sadr is in the centre of Cygnus' cross). They are close to the M 29 open cluster, but there is no indication for their membership in there. Dolidze (1961) reported a star cluster near Sadr, Dolidze 10, but we have not been able to identify it. The eight stars in the agglomerate are poorly known: there is some kind of membership information only for the four easternmost ones. The four stars (with spectral types B8 to A0 - see Table A1 were classified by Platais et al. (1998) as members of the crowded "HIP 98321" association, that occupies an extended area around the star HIP 98321 (HD 189433) and covers parts of Cepheus, Cygnus, Lyra, and Vulpecula. This association extends up to $12 \mathrm{deg}$ and is located at about $300 \mathrm{pc}$ from the Sun (Madsen, Dravins \& Lindegren 2002). Precisely, the four "HIP 98321" stars have the shortest heliocentric distances in the Escorial 34 agglomerate $(d \sim 260-370$ pc). HD 194885, with estimated spectral type A0 and an heliocentric distance of $260 \pm 20$ pc, may be an interloper of the "HIP 98321" association. The remaining four (westernmost) stars in the agglomerate, with slightly different proper motions and poorly determined Hipparcos distances, are probably background early-type stars of the Orion-Cygnus spiral arm (see, e.g., Comerón et al. [1993] for a discussion on the good visibility of the stars of this arm from the Sun position). As a result, the Escorial 34 agglomerate does not seem to be a single physical grouping of stars.

The six stars in the Escorial 35 agglomerate are members of the Lac OB1 association (Blaauw \& Morgan 1953; Hardie \& Seyfert 1959; Guetter 1976). The peculiar binary HD 213918 AB is the only Hipparcos star of the agglomerate that is not in the list of Lac OB1 members by de Zeeuw et al. (1999), but several other works support their membership in the association (Crawford 1961; Adelman 1968; Levato \& Abt 1976). It is obvious that the Escorial 35 agglomerate does not represent the whole Lac OB1 association, but a quite small fraction (de Zeeuw et al. [1999] tabulated 96 Hipparcos members of the association). Previous claims of sub-structure within Lac OB1 have been reported (see Lesh [1969], de Zeeuw et al. [1999], and references therein for a discussion on the Lac OB1 "a" and "b" subgroups). As a support for our identification, Kharchenko et al. (2005) listed four of our Hipparcos stars (actually six stars in three multiple systems, including the resolved binary 8 Lac A and B) as members of the new Galactic open cluster [KPR2005] 122 (ASCC 122). Additional studies are needed to confirm if this overdensity of early-type stars within Lac OB1 is a physical grouping or not.

\subsection{The Orion super-agglomerate}

Apart from Cep OB1, that was not so well investigated at that moment, the Ori OB1 complex was the most massive $\mathrm{OB}$ association in the $1 \mathrm{kpc}$ sphere centred on the Sun in the classical review by Blaauw (1964). It was, besides, more compact than the other massive Scorpius-Centaurus and Lac OB1 complexes. The total mass and largest overall projected diameter estimated by him for the Ori OB1 complex, at about $7600 M_{\odot}$ and $100 \mathrm{pc}$, roughly match current determinations. The currently accepted division of Ori OB1 into four subgroups was also presented by Blaauw (1964). He proposed the following sub-associations:

- Ori OB1a: north and west of the Orion Belt, 


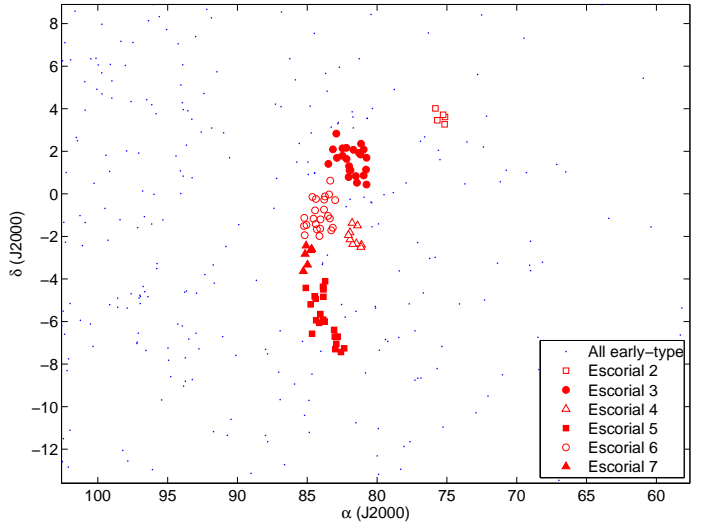

Fig. 6 Same as Fig. 5, but for the agglomerates Escorial 2-7 in the Orion super-agglomerate.

- Ori OB1b: the Orion Belt itself,

- Ori OB1c: south and east of the Orion Belt, except the Orion Sword,

- Ori OB1d: in and close to the Orion Sword.

Warren \& Hesser (1977) splitted, in its turn, the Ori OB1b subgroup into three sub-subgroups (b1, b2, and b3), and the Ori OB1c subgroup into five sub-subgroups (c, c1, c2, $\mathrm{c} 3$, and $\mathrm{c} 4$ ). The nine groupings are supposed to have different ages and heliocentric distances, in the intervals 1$10 \mathrm{Ma}$ and 350-500 pc, respectively, being Ori OB1d (including the Orion Nebula Cluster and the Trapezium) the youngest one. Further classical reviews on the Orion molecular cloud and star-forming region, emphasizing on its importance in modern Astronomy, can be found at Genzel \& Stutzki (1989) and Brown, de Geus \& de Zeeuw (1994).

Our DBSCAN algorithm has identified six agglomerates in the region $0500 \leq \alpha \leq 0540,-8 \leq \delta \leq+4$, quite close to the Solar antapex. Any blue star with low proper motion in this area is capable of belonging to the Ori OB1 complex. We consider that all but one of these agglomerations are actual members of the complex. Since they do not exactly correspond to the classical grouping in the cluster, we will follow the "Escorial" designation. See Section 4.3 for a discussion on the sub-structure of the Orion super-agglomerate.

\subsubsection{Escorial 2}

This agglomerate of only six stars, with B8-9V spectral types, is in a distinct location with respect to the other five agglomerates. Far from presenting it as a new subgroup in the Ori OB1 complex, we consider Escorial 2 a spurious agglomeration. This hypothesis is supported, apart from the different location, by: $(i)$ the low number of stars in the agglomerate (the minimum $N_{\text {MinPts }}$ ), (ii) their relatively late spectral types, (iii) the heliocentric distances shorter than $300 \mathrm{pc}$ of at least two (possibly four) stars, and (iv) the presence of two of the stars in a close binary (HD 32039, HD 32040; if they were a single star, then $N_{\star}=5<N_{\mathrm{MinPts}}$ and DBSCAN would have not recognize it as an agglomerate).

\subsubsection{Escorial 3 (25 Orionis)}

The 23 stars in the Escorial 3 agglomerate lay on the northern part of the Ori OB1a subgroup, surrounding the bright B1Vpe star 25 Ori (also known as $\psi^{01}$ Ori) ${ }^{3}$. In spite of the large amount of stars in the agglomerate, the group was not identified until very recently, when Briceño et al. (2005) and Kharchenko et al. (2005) discovered what they called the "25 Ori group" and the "ASCC 16 cluster" ([KPR2005] 16), respectively. Many of the stars in our agglomerate, including 25 Ori itself, have been catalogued in those works, which supports a correct identification. While for Briceño et al. (2005) the Escorial 3 agglomerate stood out as a concentration of T Tau stars in the Ori OB1a sub-association, Kharchenko et al. (2005) found it based on parallaxes, proper motions, and $B_{T} V_{T}$ photometry for Hipparcos and Tycho-2 stars. The confirmation of Escorial 3 having distinct kinematics (in $V_{r}$ ) and age (7-10 Ma) in Ori OB1a was presented by Briceño et al. (2007).

Not all the 23 stars belong to the young agglomerate. In Table 1, we list two possible interloper stars with heliocentric distances shorter than $200 \mathrm{pc}$. Of them, HD $35926 \mathrm{AB}$ is a binary system separated by 1.3 arcsec (Dommanget \& Nys 1994). Additionally, there are other three stars with $d+\delta d<$ $300 \mathrm{pc}$ (including errorbars) that we do not classify as possible contaminants. If we fully trust Hipparcos, HD 36429 would be a B5V star at only $d=200 \pm 30 \mathrm{pc}$. The presence of a close companion might have affected its parallax measurement (see, however, Section 4.4.4 Although Briceño et al. (2007) carried out a challenging spectroscopic study of members in Escorial 3 (25 Ori), further analyses in the agglomerate are desirable to disentangle the young star population from the fore-/background and from Ori OB1a and Ori OB1b.

\subsubsection{Escorial 4 ( $\eta$ Orionis)}

The nine stars in the Escorial 4 agglomerate are located in the southern part of the Ori OB1a subgroup, to the southwest of the Orion Belt. All of them except HD 35957, with a parallactic distance of $d=190 \pm 40 \mathrm{pc}$, seem to be members of the Ori OB1a subgroup based on early spectral types, parallaxes, low proper motions and positions in the $V_{T}$ vs. $B_{T}-V_{T}$ colour-magnitude diagram (see also Sharpless and Warren \& Hesser [1978]). To our knowledge, none of the stars have been previously reported to belong to any substructure within Ori OB1a. Since the brightest star in the

\footnotetext{
3 When available, we follow the Bayer (1603) nomenclature instead of the Flamsteed (1712) one. However, in this case there can be a misunderstanting between 25 Ori ( $\psi^{01}$ Ori) and the $\beta$ Cep-variable star $\psi$ Ori (30 Ori, $\psi^{02}$ Ori).

${ }^{4}$ HD 36429 has a previously unreported companion $(\rho \sim 9.7$ arcsec, $\theta \sim 163 \mathrm{deg}, \Delta K_{\mathrm{S}}=3.42 \pm 0.05 \mathrm{mag}$ ), apart from a known visual companion HD 287931 ( $\rho \sim 1.1 \mathrm{arcmin}, \theta \sim 350 \mathrm{deg}, \Delta K_{\mathrm{S}}=0.09 \pm 0.03 \mathrm{mag}$ ).
} 
agglomerate is $\eta$ Ori AB (28 Ori), we propose the name " $\eta$ Orionis" for the overdensity. Approximate central coordinates and diameter of the overdensity, whose hypothetical cluster nature must be confirmed, are $052630-015500$ (J2000) and $1.2 \mathrm{deg}$, respectively. From the comparison of cluster photometric sequences, the age of the stars in the $\eta$ Orionis overdensity must not differ very much from those of the 25 Ori agglomerate and the Orion Belt (e.g. 5-10 Ma).

Briceño et al. (2005) derived the basic properties ( $T_{\text {eff }}$, $L, M)$ of the seven single stars in the agglomerate, including HD 35957. Their masses ranged in the interval 2.8-9.4 $M_{\odot}$. The remaining two stars are in close binary systems, and so it is difficult to derive basic properties from photometry and low-resolution spectroscopy ( $\eta$ Ori AB, $\rho=1.695$ arcsec; HD $35456 \mathrm{AB}, \rho=0.810$ arcsec).

See Section 4.1.1 for a discussion on the possible cluster nature of the $\eta$ Orionis overdensity.

\subsubsection{Escorial 5 (Orion Sword)}

The region of the Orion Sword, including the Trapezium and the Orion Nebula Cluster, is one of the most investigated and scrutinized areas in the sky, and almost does not require any introduction (Kwan 1977; Prosser et al. 1994; Hillenbrand 1997; Lada et al. 2000; Feigelson et al. 2002). It is usually presented as "the closest region of massive star formation to the Sun" (although some authors give this honour to the Scorpius-Centaurus association). The four stars that constitute the Trapezium, the asterism that illuminates the Orion Nebula (M 42), are the brightest stars of the Orion Nebula Cluster. This cluster displays the largest stellar density in Orion (together with $\sigma$ Orionis; see below), and is considered as a distinct subgroup, Ori OB1d. Also in the Orion Sword, and running from north to south, there are other subgroups of extremely young stars that have been classified as members of the Ori OB1c1-4 sub-subgroups (Warren \& Hesser 1977). They are differentiated overdensities in the Ori OB1c subgroup. The Orion Nebula Cluster is sandwiched between Ori OB1c3 and Ori OB1c4 subsubgroups.

Practically all the 23 stars in the Escorial 5 agglomerate have been classified in the literature as members of the Ori OB1c[1-4] or Ori OB1d subgroups (e.g. Parenago 1954; Warren \& Hesser 1978). Although it is obvious that the Orion Nebula Cluster has very particular properties (e.g. very high extinction and highly concentrated radial distribution of stars), we have not been able to disentangle it from the remaining stellar population in the Orion Sword (i.e. the Ori OB1c[1-4] sub-subgroups). Indeed, $\theta^{01}$ Ori and the Trapezium stars (Hipparcos only identifies three components of $\theta^{02}$ Ori) have Tycho- $1 B_{T}-V_{T}$ colours redder than -0.05 mag and were not, therefore, in the input catalogue for our DBSCAN algorithm 5 . The brightest and bluest stars in the Escorial 5 agglomerate are $\iota$ Ori AC (O9III+, 3 mag- $V$

\footnotetext{
5 The Tycho- $2 B_{T}-V_{T}$ colour of the hierarchical triple $\theta^{01}$ Ori is, however, bluer than $-0.05 \mathrm{mag}$.
}

star in the southern border of M 42), $c$ Ori $\mathrm{AB}(\mathrm{B} 1 \mathrm{~V}+$, in the centre NGC 1977 nebula, to the north of M 42), HD 36960 (B0.5V, close to $\iota$ Ori $\mathrm{AC}$ ), and $v$ Ori (B0V, at half a degree to the south of the Trapezium).

\subsubsection{Escorial 6 (Orion Belt)}

Although the three bright O-type supergiants in the Orion Belt (Alnitak, Alnilam and Mintaka) constitute a prominent asterism in the most obvious constellation in the sky, it was not until late 1920s when Pannekoek (1929) noticed a "clustering of early-type stars elongated roughly parallel to the galactic plane". The three supergiants and dozens young stars in the Orion Belt, including $\sigma$ Orionis, have been considered since Blaauw (1964)'s work to belong to the Ori OB1b subgroup. A complete review of the (sub)stellar populations around the three supergiants is provided in Caballero \& Solano (2008). The latter authors describe the Orion Belt as the combination of: (i) a highly extinguished star-forming region surrounding Alnitak ( $\zeta$ Ori), (ii) a wide, populated, low-density cluster with no clear central overdensity surrounding Alnilam ( $\epsilon$ Ori), that is spatially coincident with the cluster Collinder 70 (Collinder 1931), and (iii) a recently-discovered cluster candidate surrounding Mintaka ( $\delta$ Ori).

The 23 stars in the Escorial 6 agglomerate have been previously considered members of the Ori OB1b subgroup (see, again, Warren \& Hesser 1978). Our list (Table A1) contains the three supergiants and other well known earlytype stars and multiple systems, like the eclipsing spectroscopic binary VV Ori AB, the likely Lindroos system 6 HD 36779 AC-B, or the helium strong, chemically peculiar, variable star V901 Ori. No possible contaminants have been identified. The blue Hipparcos stars in the vicinity of the Horsehead Nebula and the $\sigma$ Orionis cluster are not contained in the Escorial 6 agglomerate (see below).

\subsubsection{Escorial 7 (Horsehead)}

The Escorial 7 agglomerate contains seven stars, four of which were listed by Caballero (2007) among the brightest stars of the $\sigma$ Orionis cluster. Both $\sigma$ Ori AF-B and D (together with the other components of the multiple Trapezium-like system, not catalogued by Hipparcos; Caballero 2008b) are in the centre of the eponym cluster. $\mathrm{HD} 37525 \mathrm{AB}$, at 5 arcmin to the southeast of the centre, is still in the "core" of the cluster $(r \leq 20 \mathrm{arcmin}$; Caballero 2008a). The last bright cluster member, HD 37699, lies on the "halo" of the cluster, where the contamination by (overlapping) neighbouring young star populations may be large (e.g. Jeffries et al. 2006b). The B1.5V variable star HD 37744, at a bit more than 30 arcmin from the cluster centre, has occasionally been considered as a $\sigma$ Orionis member (Sherry, Walter \& Wolk 2004), but it has not been

\footnotetext{
${ }^{6}$ A Lindroos system is a binary with a main sequence, early-type primary and a post-T Tau secondary.
} 


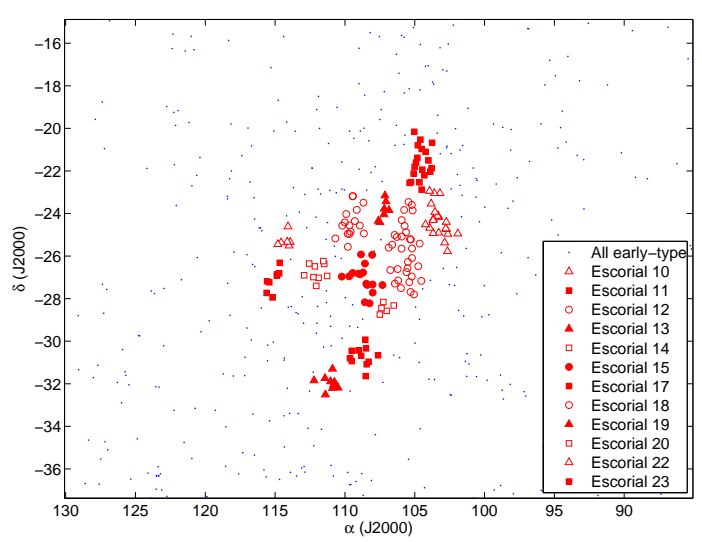

Fig. 7 Same as Fig. 5, but for the agglomerates Escorial 10-15, 17-20, 22, and 23 in the Canis Majoris-Puppis super-agglomerate. The two southern agglomerates (Escorial 17 and 19), at $\delta \leq-30 \mathrm{deg}$, belong to the Lower CMaPup grouping. The remaining agglomerates belong to the Upper CMa-Pup grouping (the Collinder 121 association).

listed in the recent Mayrit catalogue of stars and brown dwarfs in the cluster (Caballero 2008c). The other (chemically peculiar) two stars, V1148 Ori and HD 37807, are much farther away to the south. The three latter stars (HD 37744, V1148 Ori, and HD 37807) are, besides, spatially coincident with the north-to-south arrangement of the IC 434/NGC 2023 complex, whose more patent structure is the Horsehead Nebula. Because of this coincidence, and of $\sigma$ Ori illuminating the mane of the Horsehead, we call the Escorial 7 agglomerate "the Horsehead region". Increasing from $R_{\epsilon}=0.8$ to 1.0 in our DBSCAN analysis would lead the Escorial 6 (Orion Belt) and 7 (Horsehead) agglomerates to fuse into one subgroup, as commonly considered. In any case, "the Horsehead region" seems to be the juxtaposition of a highly concentrated cluster ( $\sigma$ Orionis), to the northwest, and a younger population of stars associated to the Horsehead Nebula, to the east and southeast.

\subsection{The CMa-Pup super-agglomerate}

The CMa-Pup super-agglomerate is located to the north of the Canis Majoris-Puppis-Vela region. For an easier description, we have splitted the agglomerates in CMa-Pup into two differentiated groupings: "Upper CMa-Pup" (to the north) and "Lower CMa-Pup" (to the south; Fig.7).

\subsubsection{Upper CMa-Pup (Collinder 121)}

The Upper CMa-Pup grouping is formed by the agglomerates Escorial 10-15, 18, 20, 22, and 23. Most of the stars in the agglomerates in Upper CMa-Pup except the easternmost Escorial 23 one have been classified as members of the Collinder 121 cluster/association (de Zeeuw et al. 1999; Robichon et al. 1999). According to Kaltcheva \& Makarov
(2007), Collinder 121 would be the superposition of the genuine compact group discovered by Collinder (1931), which would be at an heliocentric distance of $\sim 1 \mathrm{kpc}$, and an extended, loose OB association with a large depth probably extending from $\sim 0.5$ to beyond $1 \mathrm{kpc}$. Escorial 10 , the westernmost agglomerate in the grouping, would be the genuine Collinder 121 cluster (Feinstein 1967).

In total, ten agglomerates are associated to Collinder 121. Given its proximity to the remaining agglomerates, there is no reason to exclude the stars in Escorial 23 from the grouping. This is the first time in the literature to propose such a drastical division of Collinder 121. The extension of the region, of up to $12 \mathrm{deg}$ in both vertical and horizontal directions, would lead to maximum physical separations between stellar components of $100-200 \mathrm{pc}$. This size is not far larger than that estimated for the Orion complex (Section 3.2), which we have splitted into 5+1 agglomerates. Upper CMa-Pup/Collinder 121 might be an analog to the Orion complex, but at a triple heliocentric distance and splitted into ten agglomerates. The grouping contains the most populated agglomerate, Escorial $12\left(\mathrm{~N}_{\star}=32\right.$, roughly centred on the blue supergiant $24 \mathrm{CMa}) 7$. There are also some Upper CMa-Pup stars in known open clusters that could be associated to Collinder 121, or be in the background (e.g. NGC 2362 surrounding $\tau$ CMa AB in Escorial $18-$ age $=5_{-2}^{+1} \mathrm{Ma}, d=1480 \mathrm{pc}$; Moitinho et al. 2001). Some stars are associated to reflection nebulae (e.g. HD 61071 in Escorial 22; van den Bergh 1966).

In Table A1, we list four possible contaminants in the foreground of Upper CMa-Pup/Collinder 121. All of them have parallaxes and/or proper motions inconsistent with membership in the association (the Hipparcos measurements of $m$ Pup AB could be affected by close binarity - actually, it could be a hierarchical triple system, since the primary is thought to be a eclipsing binary; Stift 1979). It is interesting to notice the very early spectral types of some of these interlopers, like the well-known, emissionline, variable star $\omega$ CMa (B2IV/Ve - Baade 1982; Sletteback 1982; Štefl et al. 2003), which is located at only $d=$ $279 \pm 13 \mathrm{pc}$ according to van Leeuwen (2007a).

\subsubsection{Lower CMa-Pup: Escorial 17 (Collinder 132) and 19 (Collinder 140)}

There remain two agglomerates in the CMa-Pup super-agglomerate: Escorial 17 and 19. They are associated to Collinder 132 and 140, respectively, and form the Lower CMa-Pup grouping. Each of the agglomerates has only one possible foreground contaminant (Table A1).

There has been only one dedicated work in Collinder 132, carried out by Clariá (1977), and there still remain caveats on the actual nature and structure of the cluster. First, Clariá (1977) interpreted that there are two separate physical groups, called "Cr 132a" and "Cr 132b", lo-

\footnotetext{
${ }^{7}$ Star $24 \mathrm{CMa}$ is $o^{02} \mathrm{CMa}$; do not mistake with $16 \mathrm{CMa}\left(o^{01} \mathrm{CMa}\right.$, K2.5Iab:).
} 
cated at 560 and $330 \mathrm{pc}$ from the Sun, and with nuclear ages of 60 and $160 \mathrm{Ma}$, respectively. Eggen (1982) and Baumgardt (1998) supported the double cluster hypothesis, but with nuance. Baumgardt (1998) stated that "Collinder 132 is found to be mainly composed out of members of an $\mathrm{OB}$ association, but there may be a star cluster present in this area too". He also proposed that there may be a connection between Collinder 132 and Upper CMa-Pup/Collinder 121. However, Robichon et al. (1999) derived a unique parallactic distance of $d=650 \pm 140 \mathrm{pc}$, much further away than the $270 \mathrm{pc}$ tabulated by Wielen (1971), who took the value, in its turn, from Collinder (1931). Other authors have catalogued closer distances $(d \approx 400 \mathrm{pc})$ and younger ages $(\sim 25 \mathrm{Ma}$; e.g. Battinelli, Brandimarti \& Capuzzo-Dolcetta 1994).

Our Escorial 17 agglomerate possesses 11 stars. The Collinder 132 cluster centre has been considered to fall close to the brightest star in our agglomerate, HD 56342 (B3V). Indeed, this star was classified as the brightest cluster member by Clariá (1977). HD 56342 has, however, parallax and proper motion measurements clearly different from the rest of the stars of the agglomerate. This difference led us to classify it as a young contaminant star in the foreground. Obviously, an isolated bright B3V dwarf at only $d=193 \pm 8 \mathrm{pc}$ is very uncommon; it will be discussed in Section 4.4.4. From our data, we identify only one structure with heliocentric distance and age consistent with those provided by Robichon et al. (1999) $(d=650 \pm 140 \mathrm{pc})$ and Battinelli et al. (1994) (age $\sim 25 \mathrm{Ma}$ ). There might be a cluster of faint stars in the foreground, associated to HD 56342, but we fail to identify it.

Collinder 140, although discovered by Collinder (1931), could have been previously identified by de Lacaille (1755), who gave it the name "Nebulous Star Cluster No. II.2" (aka Lacaille II.2). Since Collinder 140 contains at least five stars brighter than $V=7 \mathrm{mag}$ in a concentrated arrangement, the cluster has received a larger attention than Collinder 131. Williams (1967a, 1978), Fitzgerald, Harris \& Miller (1980), Lyngå \& Wramdemark (1984), and other authors have investigated Collinder 140 in detail. Williams (1967b) suggested that Collinder 140 and NGC 2451 (Section 3.4.3), together with other two open clusters, are "the remaining nuclei of an OB association" that broke up. Clariá \& Rosenzweig (1978) carried out the most complete investigation in Collinder 140. They derived its heliocentric distance ( $d$ $=365 \pm 29 \mathrm{pc}$ ), nuclear and contraction ages (at about 20$25 \mathrm{Ma})$, physical size $(\sim 10 \mathrm{pc})$, total mass $\left(\geq 100 M_{\odot}\right)$, number of evolved members $(\geq 3)$, metallicity $([\mathrm{Fe} / \mathrm{H}]=$ -0.1 ), and other parameters, like the cluster radial velocity or the Galactic space motion. Different heliocentric distances and ages have been provided afterwards (Williams 1978: $d=420 \pm 20$ pc, age $\sim 40$ Ma; Fitzgerald et al. 1980: $d=410 \pm 30 \mathrm{pc}$, age $=20 \pm 6 \mathrm{Ma}-$ they pointed out a significant concentration of yellow giants-; Robichon et al. 1999: $\left.d=410_{-40}^{+60} \mathrm{pc}\right)$.
One of the brightest stars in Collinder 140 is the variable supergiant HD 58535 (G8II, $V=5.35 \mathrm{mag}$ - Harris 1976; Clariá 1976). It has a very red $B_{T}-V_{T}$ colour and was not in the input catalogue of our DBSCAN algorithm. The rest of the Hipparcos stars in Escorial 19 have also been classified as members of Collinder 140, except for the binary HD 59499+HD 59500 (B3V+B4V), that lies at about $1 \mathrm{deg}$ to the east of the cluster centre. We classify the binary as a Collinder 140 candidate member for the first time. Robichon et al. (1999) listed other three Hipparcos stars with $B_{T}-V_{T}>-0.05 \mathrm{mag}$ that are not, therefore, in our compilation. Besides, we have classified the EA-type eclipsing binary HD 58285 (B9III, $P=2.198515$ d, in eccentric system - Houk 1982; Otero \& Dubovsky 2004) as a possible contaminant in the outer part of Collinder 140, because of its distinguishable proper motion. The weighted mean heliocentric distance to Collinder 140, from our Hipparcos data, is $\bar{d}=370 \pm 50 \mathrm{pc}$. It is similar to the distance measured by Clariá \& Rosenzweig (1978), and consistent within errorbars with other recent determinations.

\subsection{The Pup-Vel super-agglomerate}

The Pup-Vel super-agglomerate is, as well as CMa-Pup, in the Canis Majoris-Puppis-Vela region. However, although our DBSCAN algorithm has also splitted it into ten agglomerates, the Pup-Vel super-agglomerate is not as compact as the CMa-Pup one. The ten agglomerates in Pup-Vel are distributed in a hierarchical spatial distribution. The largest spatial density coincides with a dense part of the Vel OB2 association (Section 3.4.1). Surrounding this "core", there are other seven well-defined clusters, some of which have been repeteadly investigated in the literature (IC 2391, Trumpler 10, and NGC 2451 AB).

\subsubsection{Escorial 26, 28, and 29}

Escorial 26, 28, and 29 form a dense trio of agglomerates, usually ascribed to the Vel OB2 association. The largest and most populated agglomerate is Escorial 26, that has an elongated shape in the north-south direction. Although our DBSCAN algorithm has not been able to disentangle both populations, the Escorial 26 agglomerate is actually the juxtaposition of the $\gamma$ Velorum star-forming region, to the north, and the NGC 2547 cluster, to the south. The open cluster Collinder 173 is at the northern borderline of the agglomerate.

On the one hand, the $\gamma$ Velorum region practically coincides with the densest part of the Vel OB2 association, that was identified by Kapteyn (1914) and described in detail by de Zeeuw et al. (1999) The (at least) sextuple $\gamma$ Vel star $\left(\gamma^{02} \mathrm{Vel}\right)$ is in the centre of the association, and is one of the brightest stars in the nighttime sky ( $V \approx 1.8 \mathrm{mag}$ ). The

\footnotetext{
${ }^{8}$ Do not mistake with Eggen (1982)'s Vel OB2, that corresponds to another group of stars.
} 


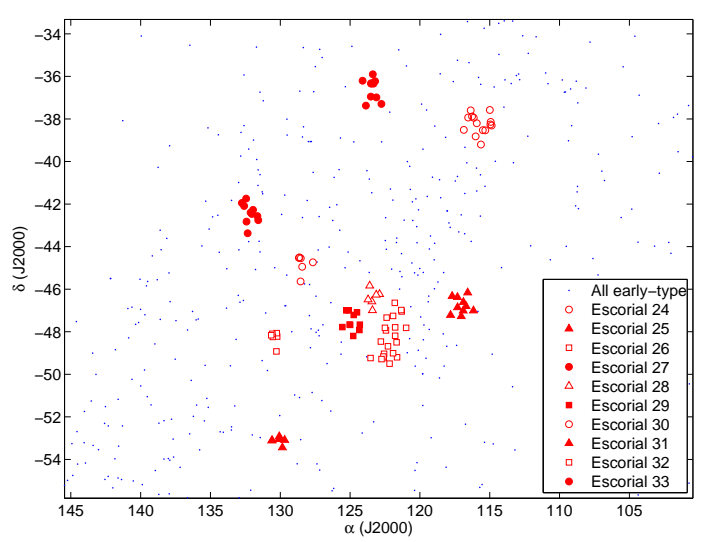

Fig. 8 Same as Fig. 5, but for the agglomerates Escorial 24-33 in the Puppis-Vela super-agglomerate. The trio Escorial 26-28 is in the centre; Escorial 30, IC 2391 (Escorial 31), IC 2395 (Escorial 32), and Trumpler 10 (Escorial 33) are to the east; NGC $2451 \mathrm{AB}$ (Escorial 24) and vdBH 23 (Escorial 27) are to the north; and Escorial 25 is to the west.

brightest component of $\gamma$ Vel is actually a spectroscopic binary composed of WR 11 (WC8), the nearest known WolfRayet star, and a massive O9I supergiant with a 78.5-day orbital period. An heliocentric distance of $411 \pm 12$ pc was derived by de Zeeuw et al. (1999). They also listed literature ages in the interval 6-20 Ma. Interestingly, the Vela Pulsar was born in Vel OB2 about $10^{4}$ a ago (Hoogerwerf, de Bruijne \& de Zeeuw 2001). On the other hand, NGC 2547 has been very well investigated. The cluster is very important for studying X-ray emission (Jeffries \& Tolley 1998; Jeffries et al. 2006a), the Wielen dip? (Naylor et al. 2002), the lithium depletion boundary (Jeffries \& Oliveira 2005), the disc frequency (Young et al. 2004; Gorlova et al. 2007), low-mass stars and brown dwarfs (Jeffries et al. 2004), mass segregation (Littlefair et al. 2003), and rotation and activity (Jeffries, Totten \& James 2000; Irwin et al. 2008). It is $30 \pm 10 \mathrm{Ma}$ old (see Oliveira et al. [2003] for a discussion on the discrepancy between ages from isochrone fitting and from the lithium depletion boundary) and at $400-450 \mathrm{pc}$, and has a low reddening (Fernie 1959, 1960; Clariá 1982 and references above). In Table1 we list three possible contaminants in the Escorial 26 agglomertae (HD 68657, at $d=$ $259 \pm 18 \mathrm{pc}$ and with a distinct proper motion, has an early spectral type B3V - Hiltner, Garrison \& Schild 1969-. It might be a B-type subdwarf in the foreground).

The other 16 stars in the trio are located to the east and northeast of Escorial 26, overlapping with the Vela supernova remnant. They are distributed among two agglomerates: Escorial 28 (northeast, $N_{\star}=6$ ) and Escorial 29 (east, $N_{\star}=10$ ). HD 70251 could be a foreground interloper in the latter agglomerate (it is a B8V stars at $d=290 \pm 30 \mathrm{pc}$

\footnotetext{
9 The Wielen dip is a feature in the luminosity function at $M_{V} \approx 7-$ 8 mag. NGC 2547 is the youngest open cluster to display it.
}

and discordant proper motion). Most of the remaining stars have been classified as members of the Vel OB2 association. Remarkably, HD 70309 A (B3III) is the primary of a Lindroos system (the secondary, HD $70309 \mathrm{~B}$, is a K2IV star Lindroos 1985; Pallavicini, Pasquini \& Randich 1992).

To sum up, we find evidence of subclustering in Vel OB2. Besides, the similarity in heliocentric distance and age between Vel OB2, $\gamma$ Velorum, and NGC 2547 may suggest that they actually belong to the same complex, but NGC 2547 having a much larger spatial density of members.

\subsubsection{Escorial 30, 31 (IC 2391), 32 (IC 2395), and 33 (Trumpler 10)}

The four agglomerates Escorial 30-33 are located to the east of the Pup-Vel super-agglomerate, rather separated between them and from the core of the Vel OB2 association formed by the trio of agglomerates described in Section 3.4.1.

Escorial 30 might be a spurious agglomeration: three of the six stars in the agglomerate have been classified as members of the concentrated, elongated Pismis 4 cluster (Moffat \& Vogt 1975; Baumgardt et al. 2000), while the other three neighbouring early-type stars seem to populate the outskirt of Pismis 4 and belong to the Vel OB2 association, or even to other open clusters in the surrounding area (e.g. HD $72350 \mathrm{AB}$ in the van den Bergh-Hagen 34 [vdBH 34] cluster; Dias et al. 2001). From our data and from Moffat \& Vogt (1975)'s, the Pismis 4 cluster is located at 500-600 pc to the Sun, in the background of the Vel OB2 association.

IC 2391 is, as well as the Pleiades, the Orion Nebula Cluster, $\sigma$ Orionis, or NGC 2547, a cornerstone for the study of the formation and evolution of stars and substellar objects at all mass domains (Hogg 1960; Perry \& Hill 1969; Stauffer et al. 1989, 1997; Patten \& Simon 1996; Barrado y Navascués, Stauffer \& Jayawardhana 2004; Boudrealt \& Bailer-Jones 2007; Siegler et al. 2007). Besides, Koester \& Reimers (1985) found a white dwarf in the cluster (IC 2391 KR 1 [WD 0839-528]), that could be the youngest one yet found, together with other white dwarfs identified towards NGC 2451 (that is described in Section 3.4.3). See also Platais et al. (2007) for recent improvements of the basic parameters of IC 2391 ( $d=159.2 \mathrm{pc}$, age $\sim 40 \mathrm{Ma}$, $[\mathrm{Fe} / \mathrm{H}]=+0.06)$. The seven stars in the Escorial 31 agglomerate are bright, classical members in the cluster. The brightest stars in IC 2391 are $o$ Vel (B3IV, $V=3.60 \mathrm{mag}$ ) and HY Vel (B3IV, $V=4.83 \mathrm{mag}$ ). In Section 4.4 .3 we derive a new heliocentric distance to the cluster.

Four of the six stars in the Escorial 32 agglomerate were classified as members of the IC 2395 (vdBH 47, Collinder 192) cluster. Of the remaining two stars in agglomerate, one (HD 74273) is well separated (by almost $1 \mathrm{deg}$ ) from the relatively concentrated cluster and may belong to a different young population in the area, while the other one (HD $74531 \mathrm{~A}$ ) has magnitudes, colours, and spectral type (B2V:) that match the IC 2395 spectro-photometric 
sequence. The cluster had been poorly investigated (Lyngå 1960, 1962; Ruprecht 1966; van der Bergh \& Hagen 1975; Jørgensen \& Westerlund 1988) until the clarifying work by Clariá et al. (2003). They derived the angular size ( $\sim 19$ arcmin), heliocentric distance $(d=800 \pm 40 \mathrm{pc})$, and age $(6 \pm 2 \mathrm{Ma})$ of IC 2395 , and suggested a physical connection to the Vel OB1c association (Mel'nik \& Efremov 1995). We can not provide additional information or improvement from our data. The brightest star in IC 2395, that may define the cluster centre, is the binary $(\rho \approx 0.3 \operatorname{arcsec})$, variable, early-type (B1.5V + mid-B) star $\mathrm{HX}$ Vel.

Six of the 10 stars in the Escorial 33 agglomerate were listed as genuine Trumpler 10 cluster members by Lyngå $(1960,1962)$, while one of the four remaining stars was also listed as a Trumpler 10 association member by de Zeeuw et al. (1999). According to the latter authors, Trumpler 10 (vbBH 53) is not "a tight open cluster, but instead an intermediate age OB association". However, the association/cluster nature of Trumpler 10 is not well understood yet. On the one hand, Lyngå tabulated a diametre of only 14 arcmin for the cluster; this value contrasts with the $\sim 8$ deg estimated by de Zeeuw et al. (1999) for the association. The doubt of the "entity" being a single cluster had been firstly proposed by Stock (1984) 10 . Although the original Lyngå's clustering of bright stars is evident in digitized plates (see Fig A11), the mean heliocentric distance to the sparse association computed by de Zeeuw et al. (1999; $d=366 \pm 23$ ) is consistent with previous determinations of distance to the cluster (Levato \& Malaroda 1975a; Eggen 1980; Lyngå \& Wramdemark 1984). The identification of the Escorial 33 agglomerate also supports the existence of an OB association of age $\sim 15 \mathrm{Ma}$ in the area, whose densest part would be the original Lyngå's cluster. Finally, Kaplan, van Kerkwijk \& Anderson (2007) measured the parallactic distance to the isolated neutron star RX J0720.4-3125 $\left(d=360_{-90}^{+170} \mathrm{pc}\right)$, and suggested an origin for it in the Trumpler 10 association $0.7_{-0.2}^{+0.2}$ Ma ago. Escorial 33/Trumpler 10 deserves further analyses on its stellar content and basic properties (age, distance, spatial distribution, mass function).

\subsubsection{Escorial 24 (NGC $2451 \mathrm{AB}$ ) and 27 (vdBH 23)}

The northernmost agglomerates in the Pup-Vel super-agglomerate, roughly equidistant from the maximum star densities of CMa-Pup and Pup-Vel, are Escorial 24 and 25 .

Escorial 24 is associated to NGC $2451 \mathrm{AB}$, which is actually the superposition of two clusters at different heliocentric distances. First catalogued by Dreyer (1888), NGC 2451 is one of the ten closest open clusters to the Sun (see Hünsch, Weidner \& Schmitt [2003] for a historical review).

\footnotetext{
10 There are additional foreground open clusters at less than 1 deg to the highest star concentration of Trumpler 10. NGC 2671 is an $\sim 80$ Ma-old cluster at $d \sim 1700 \mathrm{pc}$ (Pedreros 2000). ESO 260-8 is an ultracompact $\mathrm{H}$ II region, apart from a radio (methanol maser) and IRAS source, at $d \sim$ 1.3 kpc (Griffith \& Wright 1993; Walsh et al. 1997; Dutra et al. 2003).
}

It was firstly studied in detail (as a single cluster) by Feinstein (1966) and in a series of papers by Williams (1966, 1967b). Prior to 1990 , it was revisited by Levato \& Malaroda (1975b) and other authors (Andersen \& Reiz 1983; Eggen 1983; Pastoriza \& Ropke 1983; Maitzen \& Catalano 1986; Gilroy 1989). There have been later studies in NGC 2451 on close resolved photometry (Platais et al. 2001), photometry and membership (Carrier, Burki \& Richard 1999), X-ray emission (Hünsch et al. 2003), and rotation and lithium abundance (Hünsch et al. 2004). The "binary" status of NGC 2451 A and B has also been confirmed by many authors (Baumgardt 1998; Röser \& Bastian 1994, and references above). Robichon et al. (1999) derived $d=188.7_{-6.5}^{+7.0} \mathrm{pc}$ for NGC $2451 \mathrm{~A}$, and determined an age of 35-55 Ma, consistent within error bars with the more recent determination by Platais et al. (2001; 60土20 Ma) and Hünsch et al. (2003; 56-80 Ma). NGC 2451 A could be the core of a moving group (the Puppis Moving Group Röser \& Bastian 1994). NGC 2451 B is of a similar age but, in contrast, located further away. Carrier et al. (1999) derived $d=358 \pm 22 \mathrm{pc}$ for NGC $2451 \mathrm{~B}$. These authors also investigated in detail the exceptional emission-line star V468 Pup (B6IVe).

Our list of 14 stars in Escorial 24 approximately match other lists of bright blue stars in NGC 2451 (both A and B). However, we classify the stars in Escorial 24 in three groups:

- 10 star members in NGC $2451 \mathrm{~A}$, with mean proper motion $\left(\overline{\mu_{\alpha} \cos \delta}, \overline{\mu_{\delta}}\right)=(-21.3 \pm 1.7,+15.4 \pm 1.0) \mathrm{mas} \mathrm{a}^{-1}$ and heliocentric distance $\bar{d} \leq 200 \mathrm{pc}$ (see Section 4.4.2);

- three star members in NGC 2451 B (HD 6189911, HD 62991, and HD 63465), with mean proper motion $\left(\overline{\mu_{\alpha} \cos \delta}, \overline{\mu_{\delta}}\right)=(-10.2 \pm 0.4,+7 \pm 3) \mathrm{mas} \mathrm{a}^{-1}$ and heliocentric distance $\bar{d} 340 \pm 30 \mathrm{pc}$; and

- star V468 Pup, with $d=430 \pm 40$ and $\left(\mu_{\alpha} \cos \delta, \mu_{\delta}\right)=$ $(-0.84 \pm 0.18,-4.4 \pm 0.2)$ mas a $^{-1}$. This star, although it was considered as a NGC 2451 B member by Carrier et al. (1999), has discordant proper motion and heliocentric distance, and belongs to a third background stellar population.

To sum up, the Escorial 24 agglomeration is the superposition of at least two young open clusters. The brightest star in the area, c Pup AB (a K2.5Ib-II star in NGC 2451 B), has a colour $B_{T}-V_{T}=2.038 \pm 0.017 \mathrm{mag}$ and was not, therefore, in the input catalogue of our DBSCAN algorithm. Based on proper motion and parallax analysis, we confirm non-membership in NGC $2451 \mathrm{~A}$ or B of the bright $(V=$ 6-8 mag) stars HD 62559 (F2IV), HD 63738 (F7V), and HD 63291 (K3II-III). Besides, HD 62595 is a G7III star that probably belong to NGC $2451 \mathrm{~B}$. The four Hipparcos stars also have colours $B_{T}-V_{T}>-0.05 \mathrm{mag}$.

\footnotetext{
11 We use the Henry Draper nomenclature instead of $\mathrm{d}^{03}$ Pup, since it is easy to misunderstand with $\mathrm{d}^{02}$ Pup (HD $61878 \mathrm{AB}$ ), that belongs to NGC 2451 A.
} 
J. A. Caballero \& L. Dinis: A revisit to agglomerates of early-type Hipparcos stars

Likewise, the Escorial 27 agglomerate contains 11 stars. The two brightest ones are the B1.5-type giant MX Pup 12 and the dwarf OS Pup. The agglomerate is to the north of the NGC 2546 cluster and in southeastern vicinity of the H II region Gum 10 (Gum 1955; Rodgers, Campbell \& Whiteoak 1960). It was Westerlund (1963) who firstly indicated the existence of an OB association in the region of the longperiod cepheid RS Pup (F8Ia), that is relatively close to Escorial 27. This association, now called Pup OB3, is very wide and contains most of the stars in our agglomerate. He estimated an age of only $4 \mathrm{Ma}$, consistent with the observed spectral types and apparent magnitudes. Later, van den Bergh \& Hagen (1975) reported a not previously catalogued cluster roughly centred on OS Pup. This cluster, van den Bergh-Hagen 23 (vdBH 23), had quite uncertain parameters until was rediscovered in more recent works (Platais et al. 1998; Kharchenko et al. 2005). An heliocentric distance of $d \sim 384 \mathrm{pc}$ was estimated by Dias et al. (2001). Although not all the stars in the Escorial 27 agglomerate have been catalogued as members of $\mathrm{vdBH} 23$, they probably represent the same entity. The O9.5II supergiant HD 68450 is probably a star in the background and does not belong to Escorial 27/vdBH 23. From the weighted mean of the parallaxes of the remaining ten stars, we derive an heliocentric distance of $\bar{d}=320 \pm 30 \mathrm{pc}$, slightly lower than Dias et al. (2001)'s estimation. A dedicated study of Escorial $27 / \mathrm{vdBH} 23$ is still to be carried out.

\subsubsection{Escorial 25 (P Puppis)}

Most of the ten stars in the Escorial 25 agglomerate are poorly known. Three of them are the $\beta$ Cep variable QS Pup and the very bright giants P Pup AC and HD $63578(V=$ 4.10 and $5.22 \mathrm{mag}$, respectively). The latter star and other two ones in the agglomerate (HD 63343 and HD 63449 AB - a close double with $\rho=0.496$ arcsec) were classified as members of the Vel OB2 association by de Zeeuw et al. (1999). Apart from photometry and astrometry, there is no additional information for the remaining stars.

The region displays an evident larger surface density of early-type stars than in its surroundings, and it is located at a considerable angular separation from the Vel OB2 trio of agglomerates (Section 3.4.1). Escorial 25 probably belongs to the Pup-Vel super-agglomerate, but not to the "core" of Vel OB2. We propose for the first time the existence of an open cluster coinciding with Escorial 25, approximately centred on P Pup AC, the brightest star in the agglomerate. Therefore, we call it the "P Puppis cluster". The ten Hipparcos stars in Escorial 25/P Puppis delineate a sharp cluster sequence in the $B_{T}$ vs. $B_{T}-V_{T}$ colour-magnitude diagram. HD 63007 (B5V) has, however, proper motion and heliocentric distance slightly different from the remaining cluster member candidates; HD 63007 might be, therefore, a foreground contaminant. Accounting

\footnotetext{
12 We use the variable star name MX Pup instead of $r$ Pup, since it is easy to misunderstand with R Pup (an F9Ia supergiant in NGC 2439).
}

for the remaining nine Hipparcos stars in the cluster, if confirmed, it would be located at an heliocentric distance of $\bar{d}$ $=470 \pm 70 \mathrm{pc}$.

The only very bright, red star in the area is the evolved giant Q Pup (K0III), with heliocentric distance and proper motion inconsistent with membership in Escorial 25/P Puppis $\left(d=70 \pm 2 \mathrm{pc}, \mu>100 \mathrm{mas} \mathrm{a}^{-1}\right)$. As a result, there is no evidence of cluster stars in the Red and Asymptotic Giant Branches. The brightest star in the cluster is an early-type giant (B0III) that has not moved away from main-sequence. The same can be applied to the subgiants QS Pup and HD 63578 (B1.5IV). The next stars in brightness order are B3-5V dwarfs, that have evolutionary ages of roughly $20 \mathrm{Ma}$. We will assume a very young age of $10_{-5}^{+10}$ for the P Puppis cluster.

Further analyses, required to ascertain the nature of Escorial 25/P Puppis, will be presented in Section 4.1.2.

\section{Discussion}

\subsection{Two new clusters? $\eta$ Orionis and P Puppis}

\subsection{1 $\eta$ Orionis}

Among the 35 identified agglomerates, two of them have never been proposed to be real clusters (i.e. their stars are gravitationally bound). They are the $\eta$ Orionis overdensity and the P Puppis cluster candidate.

As already mentioned in Section 3.2.3 the centre of the $\eta$ Orionis overdensity does not coincide with the massive (double) star $\eta$ Ori AB. In order to avoid the limits of the Hipparcos catalog and our colour selection, we carried out a new Virtual Observatory analysis of the region. In particular, we loaded with Aladin all Tycho-2 stars at less than $2 \mathrm{deg}$ to the central coordinates in Section $3.2 .3(N=734)$, and selected all of them with colours $B_{T}-V_{T}<+0.25 \mathrm{mag}$ and magnitudes $V_{T}<10.50 \mathrm{mag}(N=96)$. We checked that all the selected stars have very low proper motions $(\mu \leq$ 10 mas a $^{-1}$ ) and follow a relatively narrow secuence in the $B_{T}$ vs. $B_{T}-V_{T}$ colour-magnitude diagram, consistent with its membership in Orion, and that redder non-selected stars of similar brightess have colours (and parallaxes) typical of field dwarfs in the foreground.

We counted the number of selected stars in coronae centred on both $\eta$ Ori AB and the survey area central coordinates, and did not find a clear evidence of a dense star agglomeration as has been found in other sites in Ori OB1 (like in the Orion Nebula Cluster and the $\sigma$ Orionis cluster). Besides, the elongated shape of the overdensity gets more patent with the Tycho-2 data, which contrasts with the symmetrical, radial density gradient found in well-characterized clusters (Cartwright \& Whitworth 2004; Caballero 2008a). To sum up, we confirmed that a spread overdensity of bright, early-type stars does exist in the area of $\eta$ Ori $\mathrm{AB}$ in the Ori OB1a association. However, we failed to corroborate the existence of a (gravitationally bound) cluster in that region. 

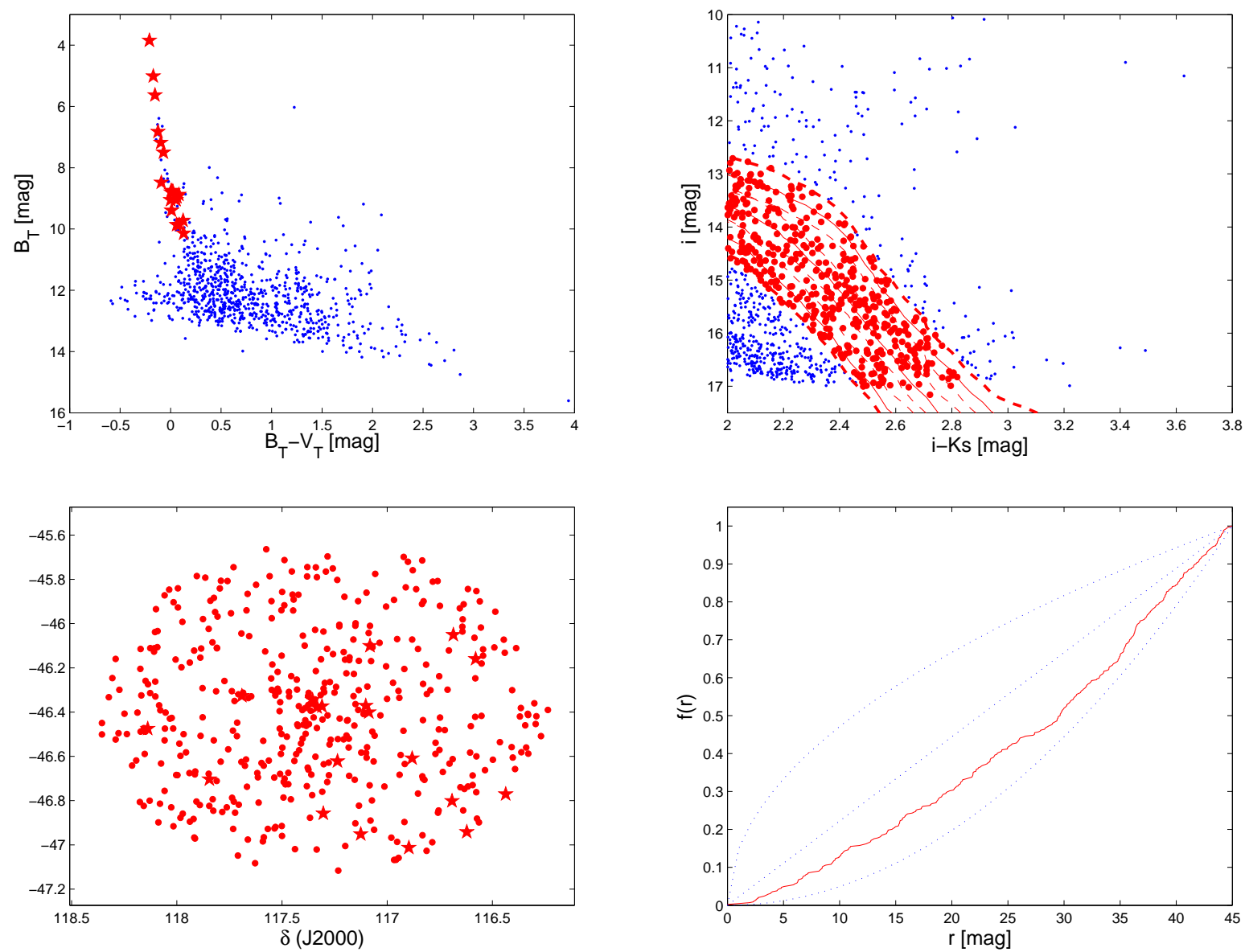

Fig. 9 Some diagrams illustrating the follow-up of the P Puppis cluster. Top left: $B_{T}$ vs. $B_{T}-V_{T}$ colour-magnitude diagram showing Tycho-2 cluster member candidates at less than 45 arcmin to P Pup AC (filled -red-stars) and remaining objects at up to 90 arcmin to the early-type binary (small -blue- dots). Top right: the faintest, reddest portion of the $i$ vs. $i-K_{\mathrm{s}}$ diagram showing DENIS/2MASS cluster member candidates at less than 45 arcmin to P Pup AC (filled -redcircles) and remaining objects at the same angular separation (small -blue- dots). Solid (red) lines are for the 5, 10, and 20 Ma-old NextGen98 isochrones (from top to bottom) shifted at $d=470 \mathrm{pc}$, while dashed (red) lines are for the previous isochroness shifted at $d=400$ (above) and $570 \mathrm{pc}$ (below). Bottom left: spatial distribution of the Tycho-2 (filled stars) and DENIS/2MASS (filled circles) cluster member candidates. Bottom right: normalized cumulative number, $f(r)$, of cluster member candidates, in solid (red) line, and theoretical power-law distributions for $f(r) \propto r^{1 / 2}, r^{1}$, and $r^{2}$ (from top to bottom). Compare with figures in Caballero (2008a).

\subsubsection{P Puppis}

We followed a similar procedure to that of the $\eta$ Orionis overdensity in the previous section for studying the possible cluster nature of the agglomerate Escorial 25/P Puppis. First, we selected all Tycho- 2 stars satisfying the colour and magnitude restrictions listed above. In this case, we only loaded the stars in a 90 arcmin-radius circle centred on $P$ Pup AC. The photometric sequence in the colour-magnitude diagram in top left panel of Fig. 9 is very sharp, as already noticed in Section 3.4.4 with the Hippar$\cos$ data. Remarkably, the three brightest stars in the agglomerate (i.e. the two resolved components in P Pup AC and the subgiant QS Pup) lie on a circle with a radius of only 45 arcmin, which subtends and area four times smaller than the 90 arcmin-radius circle. Far from expecting four times less blue, bright stars, approximately half of them are within the smaller radius (i.e. there are twice more blue bright stars in the inner circle than expected - the deviation is at the level of five times the square root of the Poissonian variance), suggesting a dense star agglomeration.

We probed the very low-mass stellar population (close to the substellar boundary) in the 45 arcmin-radius circle using the Dee Near Infrared Survey of the Southern Sky (DENIS; Epchtein et al. 1997) and the Two-Micron All Sky Survey (2MASS; Skrutskie et al. 2006) and the NextGen98 theoretical isochrones of Baraffe et al. (1998). We proceed as in Caballero \& Solano (2008), by loading DENIS and 2MASS data and cross-matching the sources. We filtered the faintest $(i>10 \mathrm{mag})$, reddest $\left(i-K_{\mathrm{S}}\right)$ sources with the 
J. A. Caballero \& L. Dinis: A revisit to agglomerates of early-type Hipparcos stars

highest quality photometry ( $\delta i, \delta J, \delta H, \delta K_{\mathrm{s}}<0.1 \mathrm{mag}$ ). Since the Virtual Observatory selection of P Puppis cluster member candidates cannot be carried out through the definition of a lower envelope of spectroscopically confirmed young stars (as in the $\sigma$ Orionis; Caballero 2008c) or of a redder envelope that both maximizes the number of cluster members and minimizes the number of contaminants (as in the Alnilam/Mintaka region; Caballero \& Solano 2008), we opted for the classical selection criterion based on theoretical isochrones. As can be seen in the colour-magnitude diagram in top left panel in window Fig. 9, the theoretical $\mathrm{P}$ Puppis sequence, considering all possible uncertainties in the cluster heliocentric distance $(d=470 \pm 70 \mathrm{pc})$ and age $\left(10_{-5}^{+10} \mathrm{Ma}\right)$, is quite broad and overlaps with the location of a lot of (foreground) field M-type dwarfs. Other colourmagnitude diagrams, performed with the same technique in regions of exactly the same area and galactic latitude, and of approximately the same galactic longitude, are quite similar, which indicates that many of the selected cluster member candidates (between the $5 \mathrm{Ma}, 400 \mathrm{pc}$ and the $20 \mathrm{Ma}, 540 \mathrm{pc}$ NextGen isochrones) are actually late-type interlopers. Although some elaborated de-contamination technique could be applied (e.g. Caballero, Burgasser \& Klement 2008), a central overdensity of selected cluster members is already obvious (see the spatial distribution of selected sources in the bottom left panel in Fig. 97. Since there are 425 DENIS/2MASS selected sources in the 45 arcmin-radius circle, one should expect $\sim 100$ such objects in the four-times smaller area defined by a 22.5 arcmin-radius circle centred on P Pup AC. In contrast, we found more than 150 of them. In other words, there are at least $\sim 50$ (young) low-mass stars at less than 20-25 arcmin to the B0 giant that probably form part of the P Puppis cluster.

Other way of illustrating the central overdensity of $P$ Puppis is the diagram of the normalized cumulative number of cluster member candidates, $f(r)=N(r) / N\left(r_{\max }\right)$, as a function of angular distance to the the centre, $r$. A wealth of details on how to compute and interpret it are in Caballero (2008a). In short, a power-law distribution $f(r) \propto$ $r^{2}$ corresponds to a uniform distribution of objects in the survey area, while $f(r) \propto r^{1}$ corresponds to a volume density proportional to $r^{-2}$, which is consistent with the collapse of an isothermal spherical molecular cloud (e.g. Burkert, Bate \& Bodenheimer 1997). A distribution with an intermediate index, as that one observed in the Mintaka cluster (Caballero \& Solano 2008) and in P Puppis from $r=$ 0 to $\sim 25$ arcmin (bottom right panel in Fig. 9) corresponds to the superposition of a radially-concentrated cluster with $f(r) \propto r^{1}$ and a homogeously-distributed population of (late-type, field dwarf) contaminants.

To sum up, we discover a star cluster surrounding the young, massive P Pup AC giant. However, a dedicated spectroscopic follow-up will be necessary to disentangle between the stellar populations of the cluster and of the field.

\subsection{New sites for substellar searches}

Some of the agglomerates identified in our work are valuable "hunting grounds" for the search of faint brown dwarfs and planetary-mass objects. In particular, the first brown dwarf, Teide 1, was found in the Pleiades cluster (Rebolo et al. 1995), while the Orion Nebula and the $\sigma$ Orionis clusters harbour the great majority of the planetary-mass objects with spectroscopy (Lucas \& Roche 2000; Zapatero Osorio et al. 2000). Other listed agglomerates that have held substellar searches are the Orion Belt (surrounding Alnilam and Mintaka), NGC 2264, NGC 2547/ $\gamma$ Velorum, and IC 2391. However, there are agglomerates in our list that share properties (youth, closeness, low extinction) that also make them ideal sites for substellar searches.

After discarding the possible spurious agglomerates and the groupings associated to Collinder 121 and Vel OB2, that are too far away and/or too sparse for efficient searches, we retained 14 agglomerates for a follow-up inspection of their parameters. Of them, five seem to be at prohibitive heliocentric distances larger than 650 pc (i.e. NGC 2353 , Collinder 132, NGC 2362, Pismis 4, and IC 2395). Besides, M 47, although being located at $d \sim 470$ pc, has a Pleiadeslike age and, therefore, their hypothetical brown dwarfs will be about 3 mag fainter.

We maintain eight agglomerates as new sites for substellar searches. Two of them are obvious choices, because they resemble their "elder brothers" (in contraposition to "fraternal twins") in Orion: 25 Orionis and $\eta$ Orionis. Even in the case that $\eta$ Orionis is not a real cluster, the large observed stellar surface density will facilitate forthcoming studies (stellar and substellar objects are expected to have proportional surface densities; Caballero 2008a). NGC $2451 \mathrm{~A}$, with an age of $\sim 45 \mathrm{Ma}$ and a very close heliocentric distance of $d=180-200 \mathrm{pc}$ (see Section 4.4.3) is other apparent option.

If compiled basic data for vdBH 23 (Escorial 27) and Trumpler 10 (Escorial 33), with distances and ages in the approximate intervals $320-360 \mathrm{pc}$ and $1-4 \mathrm{Ma}$, are correct, then they might be new cornerstones for the substellar searches in the near future. Finally, there remain in order of interest, NGC 2232 (Escorial 8; $d \sim 320 \mathrm{pc}$, age $\sim 30 \mathrm{Ma}$ ), Collinder 140 (Escorial 19; $d \sim 370 \mathrm{pc}$, age $\sim 20 \mathrm{Ma}$ ), and our new cluster P Puppis (Escorial 25; $d \sim 470 \mathrm{pc}$, age $\sim$ $10 \mathrm{Ma})$. The first two clusters in the trio seem to be IC 2391 twins at larger distances, while P Puppis resembles the stellar groupings in the Orion Belt.

\subsection{Sub-structure of super-agglomerates}

We find evidence of spatial sub-structure within classical OB associations. First, the "isolated cluster" Escorial 35 could be a physical grouping within Lac OB1 (Section 3.1.6). Secondly, our three super-agglomerates (Orion, CMa-Pup, and Pup-Vela) are, by definition, the combination of a number of individual agglomerates. There 
can be sub-sub-structure (i.e. sub-structure within the agglomerates), as described in, for example, Sections 3.1.3 (NGC 2264),3.2.5(Orion Belt), and 3.4.3 (NGC 2451 AB), but it was not our aim to detect it.

The Orion super-agglomerate is composed of five agglomerates, whose boundaries do not coincide with classical divisions within the Ori OB1 complex. The Orion Sword (Escorial 5) is the superposition of 23 stars previously ascribed to the sub-associations Ori OB1c[1-4] and Ori OB1d, and includes the brightest stars of, e.g., the Orion Nebula Cluster. The Orion Belt (Escorial 6) coincides with Ori OB1b, except for the fact that it does not contain the stars in the nearby $\sigma$ Orionis cluster, that form the largest spatial overdensity in a new agglomerate of early-type stars close to the Horsehead Nebula (Escorial 7). Finally, we identify two agglomerates surrounding the very bright stars 25 Ori (Escorial 3) and $\eta$ Ori (Escorial 4) in the Ori OB1a sub-association. While the 25 Ori agglomerate was identified very recently, we report here for the first time the existence of a possible open cluster surrounding $\eta$ Ori.

The classical view of sequential formation of subgroups in OB associations by Elmegreen \& Lada (1977) is reinforced by our results. As shown in Fig. 6, the stars in the Orion super-agglomerate are aligned in the north-south direction. Noticeably, the oldest (or less young) agglomerate is located northward (25 Ori; $\sim 10 \mathrm{Ma}$ ), the youngest one southward (Orion Sword; $\sim 1 \mathrm{Ma}$ ), and those ones with intermediate ages (Orion Belt, Horsehead, and, possibly, $\eta$ Ori) in the middle. Extrapolating backward, it might happen that the $\lambda$ Orionis cluster (to the north of the Orion super-agglomerate) is older than 25 Ori. On the other hand, the ionization and front shocks produced in the Orion Sword might also initiate in the near future another cycle of OB star formation to the south of Orion, where large concentrations of molecular gas have been detected by the IRAS satellite.

We have broken up the CMa-Pup super-agglomerate into Collinder 132 (Escorial 17) and Collinder 140 (Escorial 19) to the south and Collinder 121 to the north. Collinder 121 has been splitted, in its turn, into ten agglomerates. One of them, Escorial 10, would be the original Collinder 121 compact group that gives the name to the extended, loose OB association. The few dedicated spectrophotometric analyses in the region do not allow to conclude if there are younger and older regions within the super-agglomerate. The spatial sub-structure suggests, however, that Collinder 121 is an analog to the Ori OB1 association at about $1 \mathrm{kpc}$ to the Sun.

The Pup-Vel super-agglomerate displays a much larger spatial distribution complexity. Indeed, the large spread on heliocentric distances, from $\bar{d}=147 \pm 6$ pc of IC 2391 to the $d \sim 800$ pc of IC 2395 indicates that not all of them are physically bounded. NGC $2451 \mathrm{~A}$, at $\bar{d}=181 \pm 9 \mathrm{pc}$, would be another open cluster in the foreground of the extended Vel OB2 association. However, the remaining agglomerates in the super-agglomerate could actually have a common origin. All of them except Pismis $4(d \sim 500-$
$600 \mathrm{pc}$ ) lie in a relatively narrow interval of heliocentric distances: $(i) \mathrm{vdBH} 23$ at $\bar{d}=320 \pm 30$ pc; (ii) NGC $2451 \mathrm{~B}$ at $\bar{d}=340 \pm 30 \mathrm{pc} ;$ (iii) Trumpler 10 at $d \sim 360 \mathrm{pc}$; $(\mathrm{iv})$ $\gamma$ Velorum, the core of the Vel OB2 association, NGC 2547, and V468 Pup at $d=400-450 \mathrm{pc}$; and $(v)$ P Puppis at $\bar{d}$ $=470 \pm 70 \mathrm{pc}$. The mean age of these star clusters is about $30 \mathrm{Ma}$, with only vdBH 23 having been reported to be significatively younger (age $\sim 4 \mathrm{Ma}$ ). Although a careful investigation of the parallactic distances to the stars in these agglomerates will not be carried out until the GAIA mission is operative, the resemblance between the ages and distances of $\gamma$ Velorum and NGC 2547 (that coincide with the trio of agglomerates Escorial 26, 28, and 29) suggests that an important fraction of the Vel OB2 association is actually the superposition of different star-forming regions à la Orion.

\subsection{Early-type stars at $d \leq 200 \mathrm{pc}$}

A bit less than $10 \%$ of the investigated OB-type stars are at relatively short heliocentric distances, at $d \leq 200$ pc. Most of them belong to the nearby clusters Pleiades (Escorial 1), NGC 2451 A (Escorial 24), and IC 2391 (Escorial 31), but there are also possible contaminants in the foreground of our agglomerates (listed in last column in Table 1), and errors in the Hipparcos catalogue. On the one hand, cluster stars with accurate parallax measurement are important to derive cluster distances, and compare its spectro-photometric sequence with theoretical models. On the other hand, some of the foreground early-type stars may belong to the rare type of bright B-type dwarfs unbound to known open clusters, which are generally subject of intensive follow-ups in the literature. Next, we describe the identified early-types stars at $d \leq 200 \mathrm{pc}$.

\subsubsection{Pleiades}

Because of its importance for the "cosmic distance ladder", the disagreement on the actual heliocentric distance to the Pleiades is still an open problem in Astrophysics (see recent works by Zwahlen et al. [2004], Soderblom et al. [2005], Southworth, Maxted \& Smalley [2005], and Percival, Salaris \& Groenewegen [2005]). Far from taking back again the dispute between the main-sequence fitting and the Hipparcos distance moduli, we have computed an additional measurement of the Pleiades heliocentric distance by weight-averaging the re-reduced Hipparcos parallaxes of the ten stars in our agglomerate Escorial 1. The computed distance to the Pleiades, $\bar{d}=120 \pm 5 \mathrm{pc}$, is still shorter than the adopted value of $134-139$ pc derived from isochrone fitting, but slightly larger than the previous (probably incorrect) Hipparcos distance of 118 pc. The two Hipparcos measurements are, however, consistent with each other within error bars. There is no especial improvement in our $\bar{d}$ if we also add Asterope and Calaeno, classical Pleiads with Hipparcos parallax that were not in our input catalogue (Section 3.1.1. 


\subsubsection{NGC 2451 A}

In Section 3.4.3, we identified seven Hipparcos stars as members of the NGC 2451 A cluster. Our weight-averaged heliocentric distance to the cluster, $\bar{d}=181 \pm 9 \mathrm{pc}$, is consistent with (although slightly lower than) the value derived by Robichon et al. (1999) from the original Hipparcos data $(d$ $=188.7_{-6.5}^{+7.0} \mathrm{pc}$ ). Carrier et al. (1999) derived, however, $d=$ $197 \pm 12$ pc using Geneve photometry and isochrone fitting. Nevertheless, the difference between this value and ours, $\Delta d=16 \pm 15 \mathrm{pc}$, could be lower if state-of-the-art theoretical tracks and improved metallicity and age were used (as in Platais et al. [2007] for IC 2391 - see below). As a result, there is no hint of NGC 2451 A displaying a Pleiades-like disagreement between heliocentric and isochrone distances.

\subsubsection{IC 2391}

IC 2391 (Section 3.4.2), with an age of about $40 \mathrm{Ma}$, is probably the youngest open cluster in the solar vicinity (see, however, Barrado y Navascués et al. [2004] and references therein). Platais et al. (2007) have provided the most complete discussion on the problem of the distance to IC 2391. They derived an isochrone distance of $159.2 \mathrm{pc}$, while the classical Hipparcos distance is $d \sim 146$ pc. From the weight-average of the parallaxes of the seven stars in our Escorial 31 agglomerate, we derive a new Hipparcos distance of $\bar{d}=147 \pm 6 \mathrm{pc}$. The distance controversy is, therefore, still standing.

\subsubsection{Early-type field dwarfs}

In Table 2, we list eight foreground agglomerate contaminants at less than $d \leq 200 \mathrm{pc}$ (one binary was actually resolved by Tycho- 2 in its two components). Apart from shorter heliocentric distances than to the corresponding agglomerates, they display different proper motions and, in most cases, are located in abnormal positions in the colourmagnitude diagrams. Six of them are B6-A0 dwarfs at distances $d \approx 180-200 \mathrm{pc}$ and are not of especial interest. However, the two remaining stars stand out.

As described in Section 3.3.2. HD 56342 (B3V, $V_{r}=$ $\left.+33.4 \pm 2.8 \mathrm{~km} \mathrm{~s}^{-1}, v \sin i=26 \mathrm{~km} \mathrm{~s}^{-1}\right)$ is a contaminant of the poorly known Collinder 132 cluster. The differences between the HD 56342 and cluster heliocentric distances, $\Delta d \approx 450 \mathrm{pc}$, and proper motion, $\Delta \mu \approx 11 \mathrm{mas} \mathrm{a}^{-1}$, make unlikely its membership in Collinder 132. The star has been spectroscopically investigated in detail by Lyubimkov et al. (2002) and subsequent papers. HD 56342 has typical effective temperature, mass, surface gravity, and helium abundance for its spectral type and class. Furthermore, they derived $d=209 \pm 36$ pc based solely on spectro-photometric parameters, which is consistent with our parallactic distance $(d=193 \pm 8 \mathrm{pc})$. Savage et al. (1985) and Berghoefer, Schmitt \& Cassinelli (1996) also derived independent distances at $d \approx 240$ and $236 \mathrm{pc}$ from ultraviolet interstellar extinction with the Astronomical Netherlands Satellite and from hydrogen column density with ROSAT. With age and mass of $54 \pm 9 \mathrm{Ma}$ and $5.3 \pm 0.3 M_{\odot}$, respectively (Lyubimkov et al. 2002), HD 56342 is one of the very few early $\mathrm{B}$ dwarfs in the solar neighbourhood that do not belong to a known cluster.

The case of $\mathrm{k}^{01}$ Pup AB (CD-26 4707) is even more extreme. Its proper motion, of $\mu \sim 29$ mas a $^{-1}$, is inconsistent with membership in the Escorial 23 agglomerate, that probably forms part of the Collinder 121 complex $(d \sim$ $0.5-1.0 \mathrm{kpc}) . \mathrm{k}^{01}$ Pup AB is a binary system of B5-6 stars (HD 61555 and HD 61556: $\rho=9.913 \pm 0.003 \operatorname{arcsec}, \theta=$ $318 \mathrm{deg}, \Delta H_{P}=0.21 \pm 0.01 \mathrm{mag}$; Perryman et al. 1997) located at only $d=110 \pm 8 \mathrm{pc}$. This distance agrees with that estimated by Lindroos (1985), at $d \sim 125$ pc. He also derived a very young age, of about $12 \mathrm{Ma}$. The evolved nature of the secondary (class IV), that is a helium variable star (Rivinius et al. 2003), may indicate a slightly older age. In any case, if the Hipparcos parallactic distance is correct (binarity may have affected the parallax measurement), $\mathrm{k}^{01}$ Pup $\mathrm{AB}$ could be the closest massive very young star (age $\leq 50 \mathrm{Ma}, M \geq 5 M_{\odot}$ ), even closer than the OB-type stars in Upper Scorpius, R Coronae Australis, $\rho$ Ophiuchi, Chamaeleon I+II, and IC $2391(d=130-150 \mathrm{pc}) 13$. We are ignorant of the star-forming region where $\mathrm{k}^{01}$ Pup $\mathrm{AB}$ was born or if the binary has an associated young moving group.

\subsection{Missing agglomerates? Missing stars?}

Obviously, our clustering algorithm is not able to identify all kinds of star clusters, but is mostly sensitive to agglomerates with at least six bright blue stars separated by less than $R_{\epsilon}$ (in our case, $R_{\epsilon}=0.8 \mathrm{deg}$ ). Missing cluster types are globular (e.g. $\omega$ Cen, M 13) and super star clusters (e.g. Westerlund 1). None of their stellar components were in the input catalogue described in Section 2.2 because of their faintness or reddening in the optical $B_{T} V_{T}$ bandpasses (due, in their turn, to very large heliocentric distances, evolution towards the red giant and asymptotic giant branches, or high interstellar extinction).

Some well-known open clusters are also missing. These lacking open clusters can be divided into two classes: $(i)$ clusters with ages in the approximate interval 100-600 Ma (e.g. $\alpha$ Persei, NGC 2516, M 35, Prasepe, Hyades), and (ii) clusters with ages in the approximate interval 1-10 Ma (e.g. Serpens, MBM 12, $\rho$ Ophiuchi, IC 348, $\lambda$ Orionis, $h+\chi$ Persei). There exist also explanations for their nondetection: for example, there is no Hyades member with Hipparcos colour bluer than $B_{T}-V_{T}=0.0 \mathrm{mag}$. The brightest Hyads, Aldebaran and $\theta^{02}$ Tau, are K5III and A7III giants, while the bluest Hyad with reliable Hipparcos photometry, 68 Tau AB, is an A2IV subgiant. In other words, because of stellar evolution, the Hyades turn-off point is redwards of our colour selection criterion in Section 2.2 Some-

\footnotetext{
13 The most massive nearby star is probably $\beta$ Pic (A6V, $d=$ $19.27 \pm 0.19 \mathrm{pc}$, age $\sim 12 \mathrm{Ma}) . \mathrm{k}^{01}$ Pup $\mathrm{AB}$, roughly contemporary, would
} be five times further, but would also be much more massive. 
Table 2 Early-type field dwarfs at $d \leq 200$ pc.

\begin{tabular}{cllccc}
\hline \hline HIP & Name & $\begin{array}{l}\text { Spectral } \\
\text { type }\end{array}$ & $\begin{array}{c}d \\
{[\mathrm{pc}]}\end{array}$ & $\begin{array}{c}M_{V} \\
{[\mathrm{mag}]}\end{array}$ & $\begin{array}{c}B-V \\
{[\mathrm{mag}]}\end{array}$ \\
\hline 25469 & HD 35716 & B9 & $180 \pm 30$ & 2.21 & -0.03 \\
25592 & HD 35926 AB & B7IV+ & $180 \pm 40$ & 2.08 & -0.07 \\
25600 & HD 35957 & B8 & $190 \pm 40$ & 2.07 & -0.05 \\
35083 & HD 56342 & B3V & $193 \pm 8$ & -1.10 & -0.14 \\
37173 & m Pup A(C)B & B8IV++ & $190 \pm 9$ & -1.72 & -0.09 \\
37229 & $\mathrm{k}^{01}$ Pup A & B6V & $110 \pm 8$ & -0.79 & -0.16 \\
& $\mathrm{k}^{01}$ Pup B & B5IVn & $110 \pm 8$ & -0.58 & -0.11 \\
37304 & HD 61687 AB & B6V+ & $193 \pm 18$ & 0.34 & -0.11 \\
39716 & HD 67704 & A0V & $200 \pm 14$ & 0.45 & -0.06 \\
\hline
\end{tabular}

thing similar happens to the other $100-600$ Ma open clusters.

In contrast, the effect of the post-main sequence evolution is barely detectable in the 1-10 Ma-old open clusters. However, the early-type stars in the youngest ones (e.g. $\rho$ Ophiuchi, IC 348) also have red colours for their respective spectral types because of intra-cluster extinction (there are abundant populations of Class 0 and I objects -recently born stars surrounded by thick shells- in many of these star-forming regions). The remaining young open clusters with relatively low extinction (e.g. $\lambda$ Orionis) have looser, less abundant, early-type stellar populations than our $35 \mathrm{ag}$ glomerates. Our clustering algorithm is not sensitive, either, to the detection of sparse very young associations, like Taurus-Auriga and TW Hydrae, and low-density Galactic OB associations, like many of those listed in Garmany \& Stencel (1992).

\subsection{Effect of the parametrization on the results}

We have investigated how a different choice of parameters of the DBSCAN algorithm (in particular, $R_{\epsilon}$ ) would have affected our results. On the one hand, Table 3 shows the early-type Hipparcos stars that belong to agglomerates when we use $R_{\epsilon}=0.6 \mathrm{deg}$ instead of $0.8 \mathrm{deg}$. With the new value, there appear two basic differences: $(i)$ there are only 21 agglomerates, and $(i i)$ the agglomerates contain no more than 11 stars. For example, Escorial 1 (the Pleiades) now contains only six stars, while many other agglomerates, including the young open clusters Collinder 132, M 47, and IC 2395, are not identified at all. Besides, Escorial 3 (25 Ori) is splitted into two different sub-agglomerates (Escorial 3a and $3 \mathrm{~b}$ ), which may indicate the presence of sub-sub-structure in Ori OB1a. Finally, HIP 26727 (Mintaka), that is one of the three bright supergiants in Escorial 6 (Orion Belt), is classified now as a member of Escorial 7 (Horsehead and $\sigma$ Orionis). To sum up, $R_{\epsilon}=0.6 \mathrm{deg}$ gives an incomplete sampling of our aggregates.

On the other hand, further increasing $R_{\epsilon}$ to $1.0 \mathrm{deg}$ results in the fusion of some aggregates, the detection of some new clusters, and the growth of nearby agglomerates (Fig.4). In particular:

- 6 agglomerates remain unchanged with respect to the $R_{\epsilon}$ $=0.8 \mathrm{deg}$ parametrization (Escorial 1 [Pleiades], 2 [spurious], 4 [ $\eta$ Ori], 9 [NGC 2264], 27 [vdBH 23], and 32 [IC 2395]).

- 14 agglomerates "capture" nearby bright blue stars and increase in size. Ten of the agglomerates accrete only three or less stars. There are, however, three agglomerates that drastically grow up, capturing 8 (Escorial 3 -25 Ori-), 9 (Escorial 16 -NGC 2232-), and up to 12 bright blue stars (Escorial $26-\gamma$ Velorum and NGC 2547-).

- 15 agglomerates are fused into five larger entities (with additional capture of nearby bright blue stars). The resultant groupings are the combination of Escorial 6 and 7 (Orion Belt and Horsehead in the Orion superagglomerate), 10-15 and 17, 18 and 20, 22 and 23 (all agglomerates associated to Collinder 121 along with Collinder 132), and 28 and 29 (in the dense trio in Vel OB2; Section 3.4.1. As a result, using $R_{\epsilon}=1.0 \mathrm{deg}$, the CMa-Pup super-agglomerate is splitted into only four groupings: Collinder 140 (Escorial 19, the only agglomerate in CMa-Pup that maintain its independence) and the three fusions in Collinder 121. The new Orion division gives the same number of groupings (25 Ori, $\eta$ Ori, Orion Sword, and "Orion Belt+Horsehead"). Remarkably, the large amassment Escorial 10-15 and 17 contains 135 bright blue stars, a value that does not differ very much from the number of such stars in de Zeeuw et al. (1999)'s Collinder 121 asociation.

- 19 agglomerates are new. All of them, except three, contain six or seven stars and have surface densities that are $\pi 0.8^{2} / \pi 1.0^{2}=0.64$ times smaller than our spurious agglomerate Escorial 2. Many of them are probably spurious agglomerates as well, and they would require a careful follow-up, as that carried out for the original parametrization. Two of the remaining agglomerates lie on CMa-Pup-Vel region, with $\mathrm{N}_{\star}=8$ and 9 , and may belong to the sparse stellar population of the local spiral arm of the Galaxy (Section 3). Finally, there is a 
new isolated agglomerate with a relative large number of stellar components, $\mathrm{N}_{\star}=10$, and separated from the large overdensities in Fig. 4. It includes the B0Vp-type star $\theta$ Car, that is the brightest one of the IC 2602 open cluster (age $\sim 30 \mathrm{Ma}, d=135 \pm 9 \mathrm{pc}-$ Randich et al. 1995; Stauffer et al. 1997).

\subsection{Possible errors in the input catalogue}

Apart from the large number of Hipparcos stars without parallax determination (indicated with ellipses "..." in the $d$ column in Table A1, we have also identified two stars that suffered from systematic errors during the Hipparcos reduction. Both of them display proper motions with very large error bars and improbable distances of a few tens AU (indicated with square brackets "[ ]" in the $d$ column in Table A1. They are the binary HIP $23279+$ HIP 23287 (HD $32039+$ HD 32040, B9Vn, in Escorial 2; $d=34 \pm 9$ pc) and HIP 35503 (HD 57281 AB, B5V, in Escorial 18; $d=$ $70 \pm 30 \mathrm{pc})$. We have not taken them into account in the analysis.

Finally, it seems that there was a misunderstanding in the Bonner Durchmusterung and/or the Hipparcos catalogues between two 10-11th-magnitude stars separated by $\sim 57$ arcsec. The actual K2-type star BD+05 1825 (TYC 189-1314-1), presented in Section 2.2, is the easternmost and brightest one of the pair at passbands $V_{T} R I J H K_{\mathrm{s}}$. The westernmost star (HIP 38575, TYC-1891700-1), that is brighter than BD+05 1825 only at passband $B_{T}$, is the only Hipparcos star in the area. HIP 38575 has typical colours of late B or early A dwarfs.

\section{Summary}

We have used the DBSCAN (Density-Based Spatial Clustering of Applications with Noise) data clustering algorithm to identify spatial agglomerates ("clusters") of Hipparcos stars with colours $B_{T}-V_{T}<-0.05 \mathrm{mag}$.

A total of 35 agglomerates of early-type stars (with spectral types late $\mathrm{O}, \mathrm{B}$, and very early $\mathrm{A}$ ) have arosen from the search. They are ascribed to young open clusters and OB associations, except for a few of them whose physical grouping is uncertain and seem to be spurious detections (Escorial 2 and, possibly, Escorial 16, 30, 34, and 35). Of the remaining agglomerates, four are associated to known open clusters and dense star-forming regions (the Pleiades [Escorial 1], NGC 2264 [Escorial 9], M 47 [Escorial 21], and the poorly known NGC 2232 cluster [Escorial 8]), while 26 form three super-agglomerates (agglomerates of agglomerates).

The Orion super-agglomerate, that coincides with a large fraction of the classical Orion OB1 complex, is splitted into five agglomerates: 25 Orionis (Escorial 3), $\eta$ Orionis (Escorial 4), Orion Sword (Escorial 5), Orion Belt (Escorial 6), and Horsehead (Escorial 7). This division is atypical: the most important differences with classical divisions are the existence of a new overdensity of stars surrounding $\eta$ Ori $\mathrm{AB}$, and the membership in a population different from the Orion Belt of the $\sigma$ Orionis cluster. We also confirm the recently identified cluster around 25 Ori.

The CMa-Pup super-agglomerate is broken up into Collinder 132 (Escorial 17), Collinder 140 (Escorial 19), and Collinder 121, which is separated for the first time into ten agglomerates (Escorial 10-15, 18, 20, 22, and 23). The Pup-Vel super-agglomerate is a conglomeration of open clusters in the foreground (at $d<200$ pc: IC 2391 [Escorial 31], NGC 2451 A [Escorial 24]), background (at $d>$ 500 pc: IC 2395 [Escorial 32] and, possibly, Pismis 4 [Escorial 30]), and at the average distance to the Lac OB2 association (at $d=340-470 \mathrm{pc}$ ). The latter association may comprise different young stellar populations associated to, e.g., Trumpler 10, $\gamma$ Velorum, and NGC 2547. Many of the agglomerates discussed here need, however, careful spectrophotometric analyses.

The open cluster P Puppis, presented here for the first time, could also be a distant member of the Lac OB2 association. We have carried out a dedicated study of the cluster using Tycho-2, DENIS, and 2MASS data and theoretical isochrones of the Lyon group and quantified its radial density gradient using the normalized cumulative number of cluster member candidates.

We have listed seven agglomerates whose substellar populations will probably be investigated in the future. They are the 25 Orionis and $\eta$ Orionis overdensities in the Ori OB1a association, the nearby, $\sim 45$ Ma-old cluster NGC 2451 A, and four poorly known clusters at $d=320$ $470 \mathrm{pc}$ with younger ages (NGC 2232, P Puppis, vdBH 23, and Trumpler 10).

We have investigated in detail the early-type Hippar$\cos$ stars in agglomerates with heliocentric distances $d \leq$ $200 \mathrm{pc}$. By weight-averaging their parallaxes, we have computed new distances to the Pleiades $(\bar{d}=120 \pm 5 \mathrm{pc})$, NGC 2451 A $(\bar{d}=181 \pm 9 \mathrm{pc})$, and IC $2391(\bar{d}=147 \pm 6 \mathrm{pc})$. The dispute between the isochrone fitting and Hipparcos distances still goes on for the Pleaides and IC 2391, but there is no controversial for NGC $2451 \mathrm{~A}$. The remaining ten stars at $d \leq 200 \mathrm{pc}$ are two stars with errors in the Hipparcos parallax measurements, six B6-A0 dwarfs with typical absolute magnitudes and colours in the foreground, and two standing early/intermediate B stars. The latter stars are HD 56342 (age $\sim 54 \mathrm{Ma}, M \sim 5.3 M_{\odot}$ ), a B3V star at $d$ $=193 \pm 8 \mathrm{pc}$, and $\mathrm{k}^{01}$ Pup AB, two B5-6 dwarfs separated by $\rho \sim 1090 \mathrm{AU}$ and located at only $d=110 \pm 8 \mathrm{pc}$ to the Sun. If the Hipparcos parallax measurement is correct, then $\mathrm{k}^{01}$ Pup $\mathrm{AB}$ would be the closest massive very young star (age $\leq 50 \mathrm{Ma}, M \geq 5 M_{\odot}$ ).

Finally, we have also discussed which is the effect of the parameter choice ( $N_{\mathrm{MinPts}}$ and $R_{\epsilon}$ ) on our agglomerate identification and why some known clusters (e.g. Hyades) have not been identified.

Many of the results and hypotheses presented here will be corroborated, refined, or corrected by the future ESA 
Table 3 Agglomerates of early-type Hipparcos stars with the alternative parameters $N_{\mathrm{MinPts}}=6$ and $R_{\epsilon}=0.6 \mathrm{deg}$.

\begin{tabular}{|c|c|c|}
\hline No. & $\begin{array}{l}\text { Original } \\
\text { agglomerate } \\
\text { (Escorial) }\end{array}$ & $\begin{array}{l}\text { Hipparcos stars } \\
\text { (HIP) }\end{array}$ \\
\hline I & 1 & $17499,17527,17531,17573,17608,17702$ \\
\hline II & 2 & $23279,23287,23295,23328,23473,23508$ \\
\hline III & $3 a$ & $25163,25235,25288,25302,25340,25469$ \\
\hline IV & $3 b$ & $\begin{array}{l}25241,25378,25411,25533,25567,25582, \\
25592,25648,25655,25751,25752\end{array}$ \\
\hline $\mathrm{V}$ & 4 & $25293,25394,25480,25552,25557,25600$ \\
\hline VI & 5 & $26197,26199,26241,26314,26345,26427$ \\
\hline VII & 6 & $\begin{array}{l}26106,26213,26311,26319,26334,26405, \\
26439,26464,26508,26683\end{array}$ \\
\hline VIII & 7 & $\begin{array}{l}26549,26551,26579,26656,26694,26713, \\
{[26727]}\end{array}$ \\
\hline IX & 8 & $\begin{array}{l}30580,30660,30700,30758,30761,30772, \\
30789\end{array}$ \\
\hline $\mathrm{X}$ & 9 & $31917,31951,31955,31978,32030,32053$ \\
\hline $\mathrm{XI}$ & 10 & $\begin{array}{l}33062,33070,33087,33165,33208,33215 \text {, } \\
33276,33309,33410\end{array}$ \\
\hline XII & 11 & 33586, 33666, 33695, 33721, 33796, 33841 \\
\hline XIII & 12 & $33935,33970,34041,34048,34167,34227$ \\
\hline$\ldots$ & 13 & $\ldots$ \\
\hline$\ldots$ & 14 & $\ldots$ \\
\hline$\ldots$ & 15 & $\ldots$ \\
\hline$\ldots$ & 16 & $\ldots$ \\
\hline$\ldots$ & 17 & $\ldots$ \\
\hline XIV & 18 & $\begin{array}{l}35267,35370,35412,35461,35503,35539, \\
35597\end{array}$ \\
\hline $\mathrm{XV}$ & 19 & $\begin{array}{l}35700,35761,35795,35822,35855,35905 \text {, } \\
36045\end{array}$ \\
\hline$\ldots$ & 20 & $\ldots$ \\
\hline$\ldots$ & 21 & $\ldots$ \\
\hline$\ldots$ & 22 & $\ldots$ \\
\hline$\ldots$ & 23 & $\ldots$ \\
\hline$\ldots$ & 24 & $\ldots$ \\
\hline XVI & 25 & 37926, 37953, 38020, 38028, 38159, 38164 \\
\hline XVII & 26 & $39873,39919,40011,40016,40024,40059$ \\
\hline XVIII & 27 & $40218,40255,40268,40274,40321,40324$ \\
\hline$\ldots$ & 28 & $\ldots$ \\
\hline XIX & 29 & $40662,40742,40825,40851,40872,40921$ \\
\hline$\ldots$ & 30 & $\ldots$ \\
\hline $\mathrm{XX}$ & 31 & $\begin{array}{l}42400,42459,42504,42535,42536,42715 \text {, } \\
42726\end{array}$ \\
\hline$\ldots$ & 32 & $\ldots$ \\
\hline XXI & 33 & $\begin{array}{l}\text { 43055, 43085, 43182, 43209, 42340, 43326, } \\
43392\end{array}$ \\
\hline$\ldots$ & 34 & $\ldots$ \\
\hline$\ldots$ & 35 & $\ldots$ \\
\hline
\end{tabular}

GAIA space mission and subsequent analyses, including more sophisticated data clustering algorithms.

Acknowledgements. Partial financial support was provided by the Universidad Complutense de Madrid, the Spanish Virtual Observatory and the Spanish Ministerio Educación y Ciencia under grants AyA2005-02750, AyA2005-04286 and AyA2005-24102E of the Programa Nacional de Astronomía y Astrofísica and by the Comunidad Autónoma de Madrid under PRICIT project S0505/ESP-0237 (AstroCAM). LD also ackowledges financial support from Ministerio Educación y Ciencia through grants Consolider MOSAICO and FIS04-271. This research has made use of the SIMBAD, operated at Centre de Données astronomiques de Strasbourg, France, and the NASA's Astrophysics Data System as bibliographic service. We acknowledge the use of NASA's 
J. A. Caballero \& L. Dinis: A revisit to agglomerates of early-type Hipparcos stars

SkyView facility (http://skyview.gsfc.nasa.gov) located at NASA Goddard Space Flight Center.

\section{References}

Adelman, S. J. 1968, PASP, 80, 329

Ambartsumian, V. A. 1947, in Stellar Evolution and Astrophysics, (Armenian Acad. of Sci.)

Andersen, T. B. \& Reiz, A. 1983, A\&AS, 53, 181

Asiain, R., Figueras, F., Torra, J., \& Chen, B. 1999, A\&A, 341, 427

Baade, D. 1982, A\&A, 105, 65

Baraffe, I., Chabrier, G., Allard, F., Hauschildt, P. H. 1998, A\&A, 337,403

Barbera, M., Bocchino, F., Damiani, F., Micela, G., Sciortino, S., Favata, F., \& Harnden, F. R., Jr. 2002, A\&A, 387, 463

Barrado y Navascués, D., Stauffer, J. R., \& Jayawardhana, R. 2004, ApJ, 614, 386

Barrow, J. D., Bhavsar, S. P., \& Sonoda, D. H. 1985, MNRAS, 216,17

Battinelli, P. 1991, A\&A, 244, 69

Battinelli, P., Brandimarti, A., \& Capuzzo-Dolcetta, R. 1994, A\&AS, 104, 379

Battinelli, P., Efremov, Y., \& Magnier, E. A. 1996, A\&A, 314, 51

Baumgardt, H. 1998, A\&A, 340, 402

Baumgardt, H., Dettbarn, C., \& Wielen, R. 2000, A\&AS, 146, 251

Bayer, J. 1603, Uranometria: Omnium Asterismorum Continens Schemata, Nova Methodo Delineata, Aereis Laminis Expressa

van den Bergh, S. 1966, AJ, 71, 990

van den Bergh, S. \& Hagen, G. L. 1975, AJ, 80, 11

Berghoefer, T. W., Schmitt, J. H. M. M., \& Cassinelli, J. P. 1996, A\&AS, 118, 481

Berlind, A. A., Frieman, J., Weinberg, D. H. et al. 2006, ApJS, 167,1

Bica, E. L. D. \& Schmitt, H. R. 1995, ApJS, 101, 41

Blaha, C. \& Humphreys, R. M. 1989, AJ, 98, 1598

Blaauw, A. 1964, ARA\&A, 2, 213

Blaauw, A. \& Morgan, W. W. 1953, ApJ, 117, 256

Boudreault, S. \& Bailer-Jones, C. A. L. 2007, AN, 328, 709

Briceño, C., Calvet, N., Hernández, J., Vivas, A. K., Hartmann, L., Downes, J. J., \& Berlind, P. 2005, AJ, 129, 907

Briceño, C., Hartmann, L., Hernández, J., Calvet, N., Vivas, A. K., Furesz, G., \& Szentgyorgyi, A. 2007, ApJ, 661, 1119

Brown, A. G. A., de Geus, E. J., \& de Zeeuw, P. T. 1994, A\&A, 289, 101

de Bruijne, J. H. J. 1999, MNRAS, 310, 585

Burkert, A. Bate, M. R., Bodenheimer, P, 1997, MNRAS, 289, 497

Caballero, J. A. 2007, ApJ, 667, 520

Caballero, J. A. 2008a, MNRAS, 383, 375

Caballero, J. A. 2008b, MNRAS, 383, 750

Caballero, J. A. 2008c, A\&A, 478, 667

Caballero, J. A. \& Solano, E., A\&A, 2008, 485, 931

Caballero, J. A., Burgasser, A. J., Klement, R., A\&A, 2008, in press [eprint arXiv:0805.4480]

Carrier, F., Burki, G., \& Richard, C. 1999, A\&A, 341, 469

Chen, B., Asiain, R., Figueras, F., \& Torra, J. 1997, A\&A, 318, 29

Chereul, E., Crézé, M., \& Bienaymé, O. 1998, A\&A, 340, 384

Chereul, E., Crézé, M., \& Bienaymé, O. 1998, A\&AS, 135, 5

Clariá, J. J. 1972, A\&A, 19, 303

Clariá, J. J. 1974, AJ, 79, 1022

Clariá, J. J. 1976, IBVS, 1108, 1

Clariá, J. J. 1977, PASP, 89, 803

Clariá, J. J. 1982, A\&AS, 47, 323
Clariá, J. J., \& Rosenzweig, P. 1978, AJ, 83, 278

Clariá, J. J., Lapasset, E., Piatti, A. E., \& Ahumada, A. V. 2003, A\&A, 409, 541

Collinder, P. 1931, On structural properties of open galactic clusters and their spatial distribution, Lund: Nya Boktryckeriet (1931)

Comerón, F., Torra, J., Jordi, C., \& Gómez, A. E. 1993, A\&AS, 101, 37

Comerón, F., Torra, J., \& Gómez, A. E. 1998, A\&A, 330, 975

Crawford, D. L. 1961, ApJ, 133, 860

Dahm, S. E., \& Simon, T. 2005, AJ, 129, 829

Demers, S., Battinelli, P., Irwin, M. J., \& Kunkel, W. E. 1995, MNRAS, 274, 491

Dias, W. S., Lépine, J. R. D., \& Alessi, B. S. 2001, A\&A, 376, 441

Dias, W. S., Alessi, B. S., Moitinho, A., \& Lépine, J. R. D. 2002, A\&A, 389, 871

Dolidze, M. V. 1961, Astron. Cir., 223, 11

Dommanget, J. \& Nys, O. 1994, Com. de l'Observ. Royal de Belgique, 115,1

Dreyer, J. L. E. 1888, Mem. R. Astron. Soc., 49, 1

Dutra, C. M., Bica, E., Soares, J., \& Barbuy, B. 2003, A\&A, 400, 533

Eggen, O. J. 1980, ApJ, 238, 627

Eggen, O. J. 1982, ApJS, 50, 199

Eggen, O. J. 1983, AJ, 88, 197

Einasto, J., Klypin, A. A., Saar, E., \& Shandarin, S. F. 1984, MNRAS, 206, 529

Elmegreen, B. G. \& Lada, C. J. 1977, ApJ, 214, 725

Epchtein, N., de Batz, B., Capoani, L. et al. 1997, Msngr, 87, 27

Ester, M., Kriegel, H., Sander, J., \& Xu., X. 1996, Proc. 2nd Int. Conf. on Knowledge Discovery and Data Mining (KDD’96). AAAI Press, Menlo Park, CA, pp. 226-231, August 1996

Falin, J. L. \& Mignard, F. 1999, A\&AS, 135, 231

Feinstein, A. 1966, PASP, 78, 301

Feinstein, A. 1967, ApJ, 149, 107

Feigelson, E. D.. Broos, P., Gaffney, J. A., III, Garmire, G., Hillenbrand, L. A., Pravdo, S. H., Townsley, L., \& Tsuboi, Y. 2002, ApJ, 574, 258

Fernie, J. D. 1959, MNSSA, 18, 57

Fernie, J. D. 1960, MNSSA, 19, 120

Fitzgerald, M. P., Miller, M., \& Harris, G. L. H. 1980, MNRAS, 191, 95

Fitzgerald, M. P., Harris, G. L., \& Reed, B. C. 1990, PASP, 102, 865

Flamsteed, J. 1712, Historia Coelestis libri Duo quorum prior exhibet Catalogum Stellarum Fixarum Britannicum et Planetarum omnium Observationibus; posterior Transitus Syderum (...) $1676-1705$

Garmany, C. D. \& Stencel, R. E. 1992, A\&AS, 94, 211

Genzel, R. \& Stutzki, J. 1989, ARA\&A, 27, 41

Gilroy, K. K. 1989, 347, 835

Gorlova, N., Balog, Z., Rieke, G. H., Muzerolle, J., Su, K. Y. L., Ivanov, V. D., \& Young, E. T. 2007, ApJ, 670, 516

Gouliermis, D., Kontizas, M., Korakitis, R., Morgan, D. H., Kontizas, E., \& Dapergolas, A. 2000, AJ, 119, 1737

Griffith, M. R. \& Wright, A. E. 1993, AJ, 105, 1666

Guetter, H. H. 1976, AJ, 81, 1120

Gum, C. S. 1955, Mem. R. Astron. Soc., 67, 155

Hardie, R. H. \& Seyfert, C. K. 1959, ApJ, 129, 601

Harris, G. L. H. 1976, ApJS, 30, 451

Herschel, J. F. W. 1864, Philosophical Transactions of the Royal Society of London, 164, 1

Hillenbrand, L. A. 1997, AJ, 113, 1733 
Hiltner, W. A., Garrison, R. F., \& Schild, R. E. 1969, ApJ, 157, 313

Hoag, A. A., Johnson, H. L., Iriarte, B., Mitchell, R. I., Hallam, K. L., \& Sharpless, S. 1961, Publ. U.S. Naval Obs. Second Serie, 17,345

Høg, E., Fabricius, C., Makarov, V. V. et al. 2000, A\&A, 355, L27

Hogg, A. R. 1960, PASP, 72, 85

Hoogerwerf, R. \& Aguilar, L. A. 1999, MNRAS, 306, 394

Hoogerwerf, R., de Bruijne, J. H. J., \& de Zeeuw, P. T. 2001, A\&A, 365,49

Houk, N. 1982, Catalogue of two-dimensional spectral types for the HD stars, Vol. 3, Michigan Spectral Survey, Ann Arbor, Dep. Astron., Univ. Michigan, 3 (1982)

Huchra, J. P. \& Geller, M. J. 1982, ApJ, 257, 423

Humphreys, R. M. 1978, ApJS, 38, 309

Hünsch, M., Weidner, C., \& Schmitt, J. H. M. M. 2003, A\&A, 402, 571

Hünsch, M., Randich, S., Hempel, M., Weidner, C., \& Schmitt, J. H. M. M. 2004, A\&A, 418, 539

Irwin, J., Hodgkin, S., Aigrain, S., Bouvier, J., Hebb, L., \& Moraux, E. 2008, MNRAS, 383, 1588

Ivanov, G. R. 1996, A\&A, 305, 708

Jeffries, R. D. \& Tolley, A. J. 1998, MNRAS, 300, 331

Jeffries, R. D. \& Oliveira, J. M. 2005, MNRAS, 358, 13

Jeffries, R. D., Totten, E. J., \& James, D. J. 2000, MNRAS, 316, 950

Jeffries, R. D., Naylor, T., Devey, C. R., \& Totten, E. J. 2004, MNRAS, 351, 1401

Jeffries, R. D., Evans, P. A., Pye, J. P., \& Briggs, K. R. 2006b, MNRAS, 367, 781

Jeffries, R. D., Maxted, P. F. L., Oliveira, J. M., \& Naylor, T. 2006b, MNRAS, 371, L6

Jenkner, H. \& Maitzen, H. M. 1987, A\&AS, 71, 255

Johnson, H. L. \& Mitchell, R. I. 1958, ApJ, 128, 31

Jørgensen, U. G. \& Westerlund, B. E. 1988, A\&AS, 72, 193

Kaltcheva, N. T. \& Hilditch, R. W. 2000, MNRAS, 312, 753

Kaltcheva, N. T. \& Makarov, V. 2007, ApJ, 667, L155

Kaplan, D. L., van Kerkwijk, M. H., \& Anderson, J. 2007, ApJ, 660,1428

Kapteyn, J. C. 1914, ApJ, 40, 43

Kharchenko, N. V., Piskunov, A. E., Röser, S., Schilbach, E., \& Scholz, R.-D. 2005, A\&A, 440, 403

Koen, C. \& Eyer, L. 2002, MNRAS, 331, 45

Koester, D. \& Reimers, D. 1985, A\&A, 153, 260

Kwan, J. 1977, ApJ, 216, 713

de Lacaille, N. L. 1755, Nebulae of the Southern Sky, Observed by M. l'Abbé de la Caille, from the Cape of Good Hope. Mem. Acad. année 1755, page 194 [reprinted in an appendix to: Messier, C. 1780a. Errata important, .., Année 1783. Catalogue des Nébuleuses, page 225. Announce of the discovery of the Messsier objects M69 and M70. Connoissance des Temps for 1783 (published 1780), p. 408]

Lada, C. J., Muench, A. A., Haisch, K. E., Jr., Lada, E. A., Alves, J. F., Tollestrup, E. V., \& Willner, S. P. 2000, AJ, 120, 3162

Lamm, M. H., Bailer-Jones, C. A. L., Mundt, R., Herbst, W. \& Scholz, A. 2004, A\&A, 417, 557

van Leeuwen, F. 2007a, Hipparcos, the New Reduction of the Raw Data, Institute of Astronomy, Cambridge University, Cambridge, UK Series: Astrophysics and Space Science Library, Vol. 35020 Springer Dordrecht (2007)

van Leeuwen, F. 2007b, A\&A, 474, 653

Lesh, J. R. 1969, AJ, 74, 891

Levato, H. \& Malaroda, S. 1974, AJ, 79, 890
Levato, H. \& Malaroda, S. 1975a, PASP, 87, 173

Levato, H. \& Malaroda, S. 1975b, PASP, 87, 823

Levato, H. \& Abt, H. A. 1976, PASP, 88, 141

Lindroos, K. P. 1985, A\&AS, 60, 183

Littlefair, S. P., Naylor, T., Jeffries, R. D., Devey, C. R., \& Vine, S. 2003, MNRAS, 345, 1205

Lyngå, G. 1960, Arkiv för Astronomii, 2, 379

Lyngå, G. 1962, Arkiv för Astronomii, 3, 65

Lyngå, G., \& Wramdemark, S. 1984, A\&A, 132, 58

Lyra, W., Moitinho, A., van der Bliek, N. S., \& Alves, J. 2006, A\&A, 453, 101

Lyubimkov, L. S., Rachkovskaya, T. M., Rostopchin, S. I., \& Lambert, D. L. 2002, MNRAS, 333, 9

Maddox, S. J., Efstathiou, G., Sutherland, W. J., \& Loveday, J. 1990, MNRAS, 242, 43P

Madsen, S., Dravins, D., \& Lindegren, L. 2002, A\&A, 381, 446

Magnier, E. A., Battinelli, P., Lewin, W. H. G. et al. 1993, A\&A, 278, 36

Maitzen, H. M. \& Catalano, F. A. 1986, A\&AS, 66, 37

Maíz-Apellániz, J. 2001, AJ, 121, 2737

Martínez, V. J., Jones, B. J. T., Domínguez-Tenreiro, R., \& van de Weygaert, R. 1990, ApJ, 357, 50

Mel'nik, A. M. \& Efremov, Yu. N. 1995, Astronomy Letters, 21, 10

Merchán, M. \& Zandivarez, A. 2002, MNRAS, 335, 216

Merrill, P. W., Humason, M. L., \& Burwell, C. G. 1925, ApJ, 61, 389

Moitinho, A., Alves, J., Huélamo, N., Lada, C. J. 2001, ApJ, 563, L73

Moffat, A. F. J. \& Vogt, N. 1975, A\&AS, 20, 85

Naylor, T., Totten, E. J., Jeffries, R. D., Pozzo, M., Devey, C. R. \& Thompson, S. A. 2002, MNRAS, 335, 291

Nissen, P. E. 1988, A\&A, 199, 146

Oliveira, J. M., Jeffries, R. D., Devey, C. R., Barrado y Navascués, D., Naylor, T., Stauffer, J. R., \& Totten, E. J. 2003, MNRAS, 342,651

Otero, S. A. \& Dubovsky, P. A. 2004, IBVS, 5557, 1

Pallavicini, R., Pasquini, L., \& Randich, S. 1992, A\&A, 261, 245

Pannekoek, A. 1929, Publ. Astron. Inst. Amsterdam, 2, 63

Parenago, P. P. 1954, Trudy Gosud. Astron. Sternberga, 25, 1

Pastoriza, M. G. \& Ropke, U. O. 1983, AJ, 88, 1769

Patten, B. M. \& Simon, T. 1996, ApJS, 106, 489

Pedreros, M. H. 2000, RMxAA, 36, 13

Percival, S. M., Salaris, M., \& Groenewegen, M. A. T. 2005, A\&A, 429,887

Perry, C. L. \& Hill, G. 1969, AJ, 74, 899

Perryman, M. A. C., Lindegren, L., Kovalevsky, J. et al. 1997, A\&A, 323, L49

Pietrzyński, G., Gieren, W., Fouqué, P., \& Pont, F. 2001, A\&A, 371,497

Piskunov, A. E., Kharchenko, N. V., Röser, S., Schilbach, E., \& Scholz, R.-D. 2006, A\&A, 445, 545

Platais, I., Kozhurina-Platais, V., \& van Leeuwen, F. 1998, AJ, 116, 2423

Platais, I., Kozhurina-Platais, V., Barnes, S., Girard, T. M.., Demarque, P., van Altena, W. F., Deliyannis, C. P., \& Horch, E. 2001, AJ, 122, 1486

Platais, I., Melo, C., Mermilliod, J.-C., Kozhurina-Platais, V., Fulbright, J. P., Méndez, R. A., Altmann, M., \& Sperauskas, J. 2007, A\&A, 461, 509

Prisinzano, L., Micela, G., Sciortino, S., \& Favata, F. 2003, A\&A, 404, 927 
Prosser, C. F., Stauffer, J. R., Hartmann, L., Soderblom, D. R., Jones, B. F., Werner, M. W., \& McCaughrean, M. J. 1994, ApJ, 421,517

Pols, O. R., Cote, J., Waters, L. B. F. M., \& Heise, J. 1991, A\&A, 241,419

Press, W. H. \& Davis, M. 1982, ApJ, 259, 449

Randich, S., Schmitt, J. H. M. M., Prosser, C. F., \& Stauffer, J. R. 1995, A\&A, 300, 134

Rebolo, R., Zapatero Osorio, M. R., \& Martín, E. L. 1995, Nature, 377,129

Rebull, L. M., Makidon, R. B., Strom, S. E. et al. 2002, AJ, 123, 1528

Reylé, C. \& Robin, A. C. 2002, A\&A, 390, 491

Rivinius, Th., Stahl, O., Baade, D., \& Kaufer, A. 2003, IBVS, 5397,1

Robichon, N., Arenou, F., Mermilliod, J.-C., \& Turon, C. 1999, A\&A, 345, 471

Rodgers, A. W., Campbell, C. T., \& Whiteoak, J. B. 1960, MNRAS, 121, 103

Rojo Arellano, E., Peña, J. H., \& González, D. 1997, A\&AS, 123, 25

Röser, S. \& Bastian, U. 1994, A\&A, 285, 875

Ruprecht, J., Balázs, B., \& White, R. E. 1981, Catalog of Star Clusters and Associations, Supplement to Second Edition, ed. B. Balázs (Budapest: Akad. Kiadó)

Savage, B. D., Massa, D., Meade, M., \& Wesselius, P. R. 1985, ApJS, 59, 397

Schröder, S. E., Kaper, L., Lamers, H. J. G. L. M., Brown, A. G. A. 2004, A\&A, 428,149

Sharpless, S. 1952, ApJ, 116, 251

Sherry, W. H., Walter, F. M., \& Wolk, S. J. 2004, AJ, 128, 2316

Shobbrook, R. R. 1984, MNRAS, 211, 659

Siegler, N., Muzerolle, J., Young, E. T., Rieke, G. H., Mamajek, E. E., Trilling, D. E., Gorlova, N., Su, K. Y. L. 2007, ApJ, 654, 580

Skuljan, J., Hearnshaw, J. B., \& Cottrell, P. L. 1999, MNRAS, 308, 731

Skrutskie, M. F., Cutri, R. M., Stiening, R. et al. 2006, AJ, 131, 1163

Slettebak, A. 1982, ApJS, 50, 55

Smyth, M. J. \& Nandy, K. 1962, Publications of the Royal Observatory, Edinburgh, 3, 24

Soderblom, D. R., Jones, B. F., Balachandran, S., Stauffer, J. R., Duncan, D. K., Fedele, S. B., \& Hudon, J. D. 1993, AJ, 106, 1059

Soderblom, D. R., Nelan, E., Benedict, G. F., McArthur, B., Ramírez, I., Spiesman, W., \& Jones, B. F. 2005, AJ, 129, 1616

Southworth, J., Maxted, P. F. L., \& Smalley, B. 2005, A\&A, 429, 645

Stauffer, J. R., Hartmann, L. W., Jones, B. F., \& McNamara, B. R. 1989, ApJ, 342, 285

Stauffer, J. R., Caillault, J.-P., Gagne, M., Prosser, C. F., \& Hartmann, L. W. 1994, ApJS, 91, 625

Stauffer, J. R., Hartmann, L. W., Prosser, C. F., Randich, S., Balachandran, S., Patten, B. M., Simon, T., \& Giampapa, M. 1997, ApJ, 479, 776

Štefl, S., Baade, D., Rivinius, Th., Otero, S., Stahl, O., Budovičová, A., Kaufer, A. \& Maintz, M. 2003, A\&A, 402, 253

Stift, M. J. 1979, IBVS, 1540, 1

Stock, J. 1984, RMxAA, 9, 127

Strom, S. E., Strom, K. M., Brooke, A. L., Bregman, J., \& Yost, J. 1972, ApJ, 171, 267
Subramaniam, A. \& Bhatt, H. C. 2000, Bulletin of the Astronomical Society of India, 28, 255

Tago, E., Einasto, J., Saar, E., Tempel, E., Einasto, M., Vennik, J., \& Müller, V. 2008, A\&A, 479, 927

Turner, E. L., \& Gott, J. R., III 1976, ApJS, 32, 409

Uchihori, Y., Nagano, M., Takeda, M., Teshima, M., Lloyd-Evans, J., \& Watson, A. A. 2000, Astroparticle Physics, 13, 151

Vandenberg, D. A. \& Bridges, T. J. 1984, ApJ, 278, 679

Walker, M. F. 1956, ApJS, 2, 365

Walsh, A. J., Hyland, A. R., Robinson, G., \& Burton, M. G. 1997, MNRAS, 291, 261

Warren, W. H., Jr. \& Hesser, J. E. 1977, ApJS, 34, 115

Warren, W. H., Jr. \& Hesser, J. E. 1978, ApJS, 36, 497

Weinmann, S. M., van den Bosch, F. C., Yang, X., \& Mo, H. J. 2006, MNRAS, 366, 2

Westerlund, B. E. 1963, MNRAS, 127, 71

White, S. D. M. \& Frenk, C. S. 1991, ApJ, 379, 52

Williams, P. M. 1966, MNASSA, 25, 122

Williams, P. M. 1967a, MNASSA, 26, 126

Williams, P. M. 1967b, MNASSA, 26, 139

Williams, P. M. 1978, MNRAS, 183, 49

Young, E. T., Lada, C. J., Teixeira, P. et al. 2004, ApJS, 154, 428

Young, E. T., Teixeira, P. S., Lada, C. J. et al. 2006, ApJ, 642, 972

de Zeeuw, P. T., Hoogerwerf, R., de Bruijne, J. H. J., Brown, A. G. A., \& Blaauw, A. 1999, AJ, 117, 354

Zug, R. S. 1933, Lick Observatory bulletin, 16, 119

Zwahlen, N., North, P., Debernardi, Y., Eyer, L., Galland, F., Groenewegen, M. A. T., \& Hummel, C. A. 2004, A\&A, 425, L45

\section{A Early-type Hipparcos stars in agglomerates}

NOTE to the reader: Figs. A1 to A12 and the complete Table A1 will appear in the on-line version of Astronomische Nachrichten. 
Fig. A1 False-colour DSS-2 images of the agglomerates of early-type Hipparcos stars centred on reference stars in Table 1. Blue, green, and red are for photographic $B_{J}, R_{F}$, and $I_{N}$. North is up, east is left. All the images are $3 \times 3$ deg ${ }^{2}$ in size. Fits images were obtained from SkyView and combined with DS9. From left to right: Escorial 1 (Pleiades), 2 , and 3 (25 Ori).

Fig. A2 Same as Fig. A1, but for Escorial 4 ( $\eta$ Ori; left), Escorial 5 (Orion Sword; centre) and Escorial 6 (Orion Belt; right).

Fig. A3 Same as Fig. A1 but for the agglomerates Escorial 7 (Horsehead; left), Escorial 8 (NGC 2232; centre), and Escorial 9 (NGC 2264; right).

Fig. A4 Same as Fig. A1 but for the agglomerates Escorial 10, 11, and 12, from left to right. They are associated to Collinder 121.

Fig. A5 Same as Fig. A1 but for the agglomerates Escorial 13, 14, and 15, from left to right. They are also associated to Collinder 121 .

Fig. A6 Same as Fig. A1, but for the agglomerates Escorial 16 (including NGC 2353; left), Escorial 17 (Collinder 132 ; centre), and Escorial 18 (associated to Collinder 121; right).

Fig. A7 Same as Fig. A1, but for the agglomerates Escorial 19 (Collinder 140; left), Escorial 20 (associated to Collinder 121; centre), and Escorial 21 (M 47; right).

Fig. A8 Same as Fig. A1 but for the agglomerates Escorial 22 and 23 (associated to Collinder 121) and Escorial 24 (NGC 2451 AB; right).

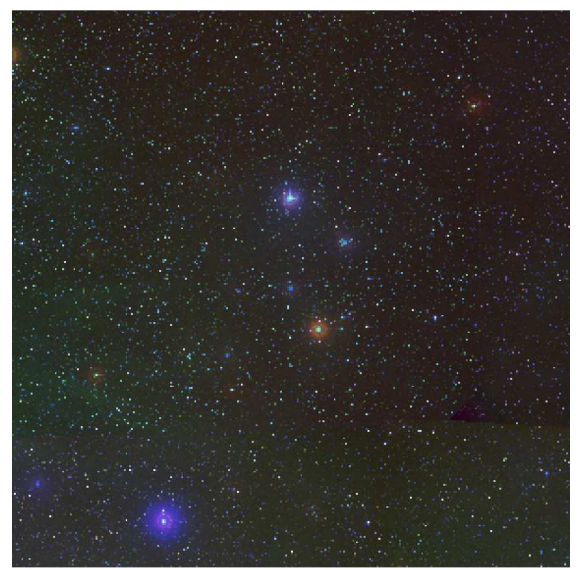

Fig. A9 Same as Fig. A1 but for the agglomerates Escorial 25 (P Puppis; left - NOTE to the reader: this is the only agglomerate to be plotted), Escorial 26 (associated to Vel OB2, and including NGC 2547 and $\gamma$ Velorum; centre), and Escorial 27 (vdBH 23; right).

Fig. A10 Same as Fig. A1, but for the agglomerates Escorial 28 and 29 (associated to Vel OB2), and Escorial 30 (including vdBH 34 and Pismis 4; right).

Fig. A11 Same as Fig. A1 but for the agglomerates Escorial 31 (IC 2391; left), Escorial 32 (IC 2395; centre), and Escorial 33 (Trumpler 10; right).

Fig. A12 Same as Fig. A1, but for the agglomerates Escorial 34 and 35. 
Table A1 Early-type Hipparcos stars in aggregates (PARTLY shown).

\begin{tabular}{|c|c|c|c|c|c|c|c|c|}
\hline Agglomerate & HIP & Name & $\begin{array}{l}\text { Spectral } \\
\text { type }\end{array}$ & $\begin{array}{c}d \\
{[\mathrm{pc}]}\end{array}$ & $\begin{array}{c}\alpha \\
(\mathrm{J} 2000)\end{array}$ & $\begin{array}{c}\delta \\
(\mathrm{J} 2000)\end{array}$ & $\begin{array}{c}\mu_{\alpha} \cos \delta \\
{\left[\mathrm{mas} \mathrm{a}^{-1}\right]}\end{array}$ & $\begin{array}{c}\mu_{\delta} \\
{\left[\mathrm{mas} \mathrm{a}^{-1}\right]}\end{array}$ \\
\hline \multirow[t]{10}{*}{ Escorial 1} & 17499 & Electra & B6IIIe & $124 \pm 4$ & 034452.54 & +240648.0 & $+20.9 \pm 0.3$ & $-46.0 \pm 0.2$ \\
\hline & 17527 & $18 \mathrm{Tau}$ & B8V & $125 \pm 6$ & 034509.74 & +245021.3 & $+20.4 \pm 0.4$ & $-46.5 \pm 0.4$ \\
\hline & 17531 & Taygeta & B6IV & $125 \pm 5$ & 034512.49 & +242802.2 & $+21.3 \pm 0.4$ & $-40.5 \pm 0.4$ \\
\hline & 17573 & Maia & B8III & $117 \pm 4$ & 034549.61 & +242203.9 & $+21.2 \pm 0.4$ & $-46.0 \pm 0.3$ \\
\hline & 17608 & Merope & B6IVe & $116 \pm 5$ & 034619.57 & +235654.1 & $+21.2 \pm 0.4$ & $-43.6 \pm 0.3$ \\
\hline & 17702 & Alcyone & B7IIIe & $124 \pm 6$ & 034729.08 & +240618.5 & $+19.3 \pm 0.4$ & $-43.7 \pm 0.3$ \\
\hline & 17776 & HD 23753 & $\mathrm{~B} 8 \mathrm{~V}$ & $118 \pm 5$ & 034820.82 & +232516.5 & $+18.0 \pm 0.4$ & $-46.6 \pm 0.3$ \\
\hline & 17847 & Atlas AB & B8III+ & $117 \pm 5$ & 034909.74 & +240312.3 & $+17.7 \pm 0.4$ & $-44.2 \pm 0.3$ \\
\hline & 17851 & Pleione & B8IVev & $117 \pm 4$ & 034911.22 & +240812.2 & $+18.1 \pm 0.3$ & $-47.2 \pm 0.3$ \\
\hline & 17900 & HD 23923 & B8V & $115 \pm 8$ & 034943.53 & +234242.7 & $+16.7 \pm 0.6$ & $-44.8 \pm 0.5$ \\
\hline \multirow[t]{6}{*}{ Escorial 2} & 23279 & HD 32039 & B9Vn & $\ldots$ & 050032.54 & +033653.3 & $(+10.5 \pm 1.3)$ & $(-18.2 \pm 1.3)$ \\
\hline & 23287 & HD 32040 & B9Vn & {$[34 \pm 9]$} & 050033.93 & +033656.7 & $(+13.0 \pm 1.4)$ & $(-16.1 \pm 1.3)$ \\
\hline & 23295 & HD 32056 & B9 & $220 \pm 70$ & 050039.79 & +031555.2 & $+0.4 \pm 1.3$ & $-4.6 \pm 0.8$ \\
\hline & 23328 & V1360 Ori & $\mathrm{B} 8 \mathrm{~V}$ & $260 \pm 40$ & 050106.02 & +034302.4 & $-2.0 \pm 0.7$ & $-6.7 \pm 0.4$ \\
\hline & 23473 & HD 32359 & B9V & $500 \pm 200$ & 050244.55 & +03 2727.7 & $+2.5 \pm 0.8$ & $-3.8 \pm 0.6$ \\
\hline & 23508 & HD 32431 & $\mathrm{~B} 8 \mathrm{~V}$ & $\ldots$ & $0503 \quad 17.10$ & +040056.0 & $+0.5 \pm 1.0$ & $+3.8 \pm 0.7$ \\
\hline \multirow[t]{23}{*}{ Escorial 3} & 25163 & HD 35177 & B9 & $350 \pm 150$ & 052301.93 & +014148.9 & $-1.0 \pm 1.2$ & $-1.1 \pm 0.6$ \\
\hline & 25164 & HD 35194 & B9 & $270 \pm 70$ & 052301.96 & +002620.6 & $-1.3 \pm 0.9$ & $-3.0 \pm 0.6$ \\
\hline & 25179 & HD 35203 & $\mathrm{~B} 6 \mathrm{~V}$ & $320 \pm 90$ & 052310.15 & +010822.6 & $-2.7 \pm 1.0$ & $-3.2 \pm 0.5$ \\
\hline & 25235 & V1156 Ori & $\mathrm{B} 3 \mathrm{Vw}$ & $\ldots$ & 052350.36 & +020455.8 & $+0.4 \pm 1.0$ & $+0.9 \pm 0.5$ \\
\hline & 25241 & HD 35305 & B6.5IV/V & $380 \pm 130$ & 052351.38 & +005146.3 & $+3.1 \pm 0.9$ & $-0.8 \pm 0.5$ \\
\hline & 25288 & HD 35407 & B4IVn & $470 \pm 160$ & 052436.10 & +022111.4 & $-0.2 \pm 0.7$ & $-0.9 \pm 0.4$ \\
\hline & 25302 & 25 Ori & B1Vpe & $320 \pm 90$ & 052444.83 & +015047.2 & $+0.2 \pm 0.7$ & $-0.2 \pm 0.4$ \\
\hline & 25340 & $\mathrm{HD} 35501 \mathrm{AB}$ & $\mathrm{B} 8 \mathrm{~V}+$ & $\ldots$ & 052511.40 & +015524.0 & $+1.0 \pm 1.1$ & $-2.9 \pm 0.5$ \\
\hline & 25378 & HD $35588 \mathrm{AB}$ & $\mathrm{B} 2.5 \mathrm{~V}+$ & $380 \pm 60$ & 052547.02 & +003112.9 & $+1.2 \pm 0.4$ & $-0.8 \pm 0.2$ \\
\hline & 25411 & HD 35612 & B8 & $320 \pm 100$ & 052606.00 & +005002.4 & $+2.0 \pm 0.9$ & $-1.8 \pm 0.5$ \\
\hline & 25469 & HD 35716 & B9 & $180 \pm 30$ & 052648.11 & +020405.9 & $-2.3 \pm 1.1$ & $+0.1 \pm 0.5$ \\
\hline & 25533 & HD $35834 \mathrm{AB}$ & $\mathrm{B} 8 \mathrm{~V}+$ & $370 \pm 150$ & 052736.88 & +010627.3 & $-0.2 \pm 1.1$ & $+2.1 \pm 0.5$ \\
\hline & 25567 & HD 35881 & B8V & $210 \pm 50$ & 052754.23 & +010618.2 & $+1.0 \pm 1.0$ & $-0.2 \pm 0.5$ \\
\hline & 25582 & HD 35912 & $\mathrm{~B} 2 \mathrm{~V}$ & $400 \pm 90$ & 052801.47 & +011753.7 & $-0.9 \pm 0.6$ & $+0.7 \pm 0.3$ \\
\hline & 25592 & HD $35926 \mathrm{AB}$ & B7IV+ & $180 \pm 40$ & 052810.12 & +004714.0 & $-1.7 \pm 1.3$ & $-3.2 \pm 0.6$ \\
\hline & 25648 & HD 36013 & B3V:n & $320 \pm 60$ & 052845.29 & +013838.2 & $-1.0 \pm 0.6$ & $-0.2 \pm 0.3$ \\
\hline & 25655 & V1372 Ori & B5Vne & $390 \pm 90$ & 052848.46 & +020953.0 & $-1.7 \pm 0.5$ & $-0.6 \pm 0.3$ \\
\hline & 25751 & HD 36166 & $\mathrm{~B} 2 \mathrm{~V}$ & $440 \pm 70$ & 052954.77 & +014721.3 & $+1.3 \pm 0.4$ & $-1.33 \pm 0.18$ \\
\hline & 25752 & HD 36165 & B7V & $230 \pm 50$ & 052955.55 & +020831.8 & $-2.0 \pm 0.8$ & $+0.4 \pm 0.4$ \\
\hline & 25881 & HD 36392 & $\mathrm{~B} 3 \mathrm{~V}$ & $250 \pm 60$ & 053129.89 & +014124.1 & $-1.9 \pm 1.2$ & $+0.1 \pm 0.5$ \\
\hline & 25897 & HD 36429 & $\mathrm{~B} 5 \mathrm{~V}$ & $200 \pm 30$ & 053141.22 & +024958.2 & $+2.6 \pm 0.8$ & $-1.3 \pm 0.4$ \\
\hline & 25979 & HD 36549 & B6Vwp & $320 \pm 130$ & 053239.49 & +020531.8 & $+3.5 \pm 1.3$ & $-0.9 \pm 0.5$ \\
\hline & 26098 & HD 36741 & $\mathrm{~B} 2 \mathrm{~V}$ & $340 \pm 60$ & 053357.59 & +012427.5 & $+2.8 \pm 0.6$ & $-0.6 \pm 0.2$ \\
\hline \multirow[t]{2}{*}{$\ldots$} & $\cdots$ & $\cdots$ & $\cdots$ & $\ldots$ & $\cdots$ & $\ldots$ & $\cdots$ & $\ldots$ \\
\hline & $\cdots$ & $\cdots$ & $\cdots$ & $\ldots$ & $\ldots$ & $\ldots$ & $\ldots$ & $\ldots$ \\
\hline
\end{tabular}

